\title{
Undergraduate Engineering Research Day 2021
}

$10.17975 /$ sfi-2021-005

The University of Toronto's Undergraduate Engineering Research Day (UnERD) is an annual conference aimed at providing an opportunity for undergraduate engineering students to showcase their research to industry professionals and fellow students, inspiring the exchange of innovative solutions across a wide breadth of global challenges.

STEM Fellowship came together with the UnERD organizers to provide a unique opportunity to the engineering students to publish their work in our STEM Fellowship Journal. For all the variation between project themes, it remains that all submissions are of incredibly high quality. Every abstract is demonstrative of immense creativity and high potential on the respective team's part.

On behalf of STEM Fellowship, I would like to extend my heartfelt congratulations to all students who participated in UnERD, and I wish them all the best for their future endeavours in research and engineering. It has been a privilege for us to witness the research capabilities of the next generation of students firsthand, and I am certain all entrants will continue to demonstrate excellence in their respective research careers.

\author{
Abhinand Thaivalappil \& Ruijia Zhang \\ Co-Managing Editors \\ STEM Fellowship Journal
}

\section{Dr. Sacha Noukhovitch}

Founder, President and Editor-in-Chief

STEM Fellowship/STEM Fellowship Journal

\section{Disclaimer}

These abstracts are provided for all student teams that have submitted to UnERD 2021. The STEM Fellowship Journal editorial board has made every effort to ensure proof and English editing of these abstracts in a limited amount of time, and neither organization as a whole or any of its volunteer members can be held accountable for inaccuracies that may have occurred in the abstract publication. Abstracts are published in no particular order.

\section{Acknowledgements}

We would like to thank the following members of our planning committee: Isabella Ferrara and Samir Khaki (Co-chairs), Vivek Dhande (Marketing Director), Melissa Ma (Outreach \& Sponsorships Director), Jason Sunardi (Communications Director), Erin Lin and Zainab Ali (Logistics Directors), Victoria Majolagbe and Janishan Jeyarajah (Programming Directors), Sue Kim (Judging \& Awards Director), and Edison Guo (Finance Director). This event would not have been possible without the following judges: Mechanical Engineering: Prof. James Davis, Prof. Markus Bssmann, Mr. Cameron Forbrigger. Computer Engineering: Prof. Raviraj Adve. Chemical Engineering: Mr. Tao Li. Biomedical Engineering: Mr. Mehdy Dousty, Ms. Aliaa Gouda, Prof. Dawn Kilkenny, Mr. Wayne Ngo, Ms. Meng-fen Tsai. Civil Engineering: Mr. Aly Abdelaziz. Electrical Engineering: Ms. Shiva Akbari, Mr. Mustafa Ammous, Prof. Wai tung Ng, Prof. Jorg Liebeherr, Mr. Shervin Mehryar, Mr. Tahsin Rahman Prof. Ervin Sejdic. Material Science Engineering: Mr. Abu Anand, Mr. Antoine Dumont. UTIAS: Prof. D'Eleuterio. ECP: Prof. Ken Tallman, Prof. Alan Chong, Prof. Peter Latka, Prof. Robert Irish, Prof. Deborah Tihanyi, Dr. Lydia Wilkinson. We would like to thank the follow advisors and speakers: Minh-tam Nguyen (staff advisor), Cori Hanson (faculty advisor), Prof. Omar Khan (keynote speaker), Prof. Aimy Bazylak (vice- dean, undergraduate). Our deepest gratitude towards the generous support of the Mechanical \& Industrial Engineering Department of the University of Toronto, the Edward S. Rogers Sr. Department of Electrical \& Computer Engineering of the University of Toronto, 


\section{Characterization of Dust-Related Air Pollution Events and Impact of Petroleum Coke Stockpi- les in the Athabasca Oil Sands Region, Alberta}

Chaitanya Ahuja ${ }^{1,2}$, Jeffrey R. Brook ${ }^{2}$

${ }^{1}$ Department of Chemical Engineering and Applied Chemistry, University of Toronto

2 Dalla Lana School of Public Health, University of Toronto

The Athabasca oil sands region (AOSR) in northern Alberta, Canada, holds the third-largest crude oil deposit in the world. Bitumen extraction and upgrading has a number of impacts on the ecology and communities in the region, including the production of gaseous and particulate air pollution. One source of particulate air pollution are open stockpiles of petroleum coke (petcoke) which are susceptible to wind erosion and transport as dust. Petcoke contains polycyclic aromatic hydrocarbons (PAHs), known mutagens and carcinogens, and is a major source of PAHs to the region. In order to improve management of dust produced by the oil sands industry, and petcoke stockpiles especially, the available air quality data measured in the region by local and national programs are utilized to identify and characterize pollution events with high dust influence, and evaluate the hypothesized influence of petcoke on these events. This data includes hourly meteorological and basic pollutant concentration measurements, 24-hr particulate matter mass and elemental composition data, and 30-min detailed particulate matter size distributions with a greater number of pollutant concentrations. A framework for identifying dust events and petcoke influence within these events is developed. Statistical tools including correlations, multivariate linear regressions, and cluster analysis are applied to gain insights on seasonal and meteorological influences, co-existing pollutants, and characteristic elemental composition fingerprints of the particulate matter in dust events. Dust measurement and management practices utilized in other jurisdictions are reviewed and strategies that may be effective in the AOSR based on the analysis of dust events are proposed.

Development of Liposomes for the Encapsulation of Iron to Fortify Hibiscus sabdariffa Calyces Beverage

Erin $\mathrm{Ng}^{1}$, Ade Oyewole ${ }^{1,2}$, Levente Diosady ${ }^{1,2}$
1 Department of Chemical Engineering and Applied Chemistry, University of Toronto

${ }^{2}$ BioZone-Centre for Applied Bioscience and Bioengineering

Globally, 1.74 billion individuals are affected by anemia, with dietary iron deficiency (ID) being the main contributor. ID is prevalent in low-and middle-income countries, impacting the health of the population and stunting socio-economic development. Recently, Hibiscus sabdariffa calyces beverage is being considered as a food vehicle for iron fortification in Sub-Saharan Africa, where it is regularly consumed. However, the beverage contains polyphenols, which inhibit iron absorption by forming complexes with iron. This study aims to adopt liposomal encapsulation technology to prevent added iron from interacting with polyphenols in the beverage. Preliminary experiments focused on material selection to develop stable liposomes. Two soy lecithin sources, Acros (granular) and ADM (liquid), and their solubilities in $95 \%$ and absolute ethanol were compared. Next, the molar ratio of lecithin to cholesterol, a membrane stabilizer, was varied: 1:0, 1:.25, 1:.5, and 1:1. Liposomes were prepared by the thin film hydration method. Ethanol was removed using a rotary evaporator, the resulting thin films were rehydrated with Milli-Q water and particle sizes were reduced via ultrasonication. The liposomal suspension was separated using an ultracentrifuge. The supernatant and pellet were stored at $4^{\circ} \mathrm{C}$ pending further analysis. Liposomal suspensions prepared with granular lecithin and $95 \%$ ethanol appeared most translucent after sonication, an indicator of size reduction. However, samples prepared with cholesterol did not visibly change in opacity. Further work, including morphological and encapsulation efficiency analyses, will be required after iron loading to determine the feasibility of fortifying Hibiscus beverage with the formulated liposomes.

\section{Myoelectric interface development platform for individuals with spinal cord injury}

Xinyi Chen ${ }^{2,4}$, Jose Zariffa ${ }^{1,2,3,4}$, Gustavo Balbinot ${ }^{2}$

${ }^{1}$ KITE-Toronto Rehabilitation Institute, University Health Network

${ }^{2}$ Institute of Biomedical Engineering, University of Toronto

${ }^{3}$ Rehabilitation Sciences Institute, University of Toronto

${ }^{4}$ Edward S. Rogers Sr. Department of ECE, University of Toronto 
Cervical spinal cord injury $(\mathrm{SCl})$ results in the loss of muscle control in the upper limb, making activities of daily living challenging. Assistive technologies, such as wearable hand exoskeletons and implanted functional electrical stimulation (FES) systems, have been developed to improve independence after injury. As these technologies evolve to include more complex movement patterns, more sophisticated control signals that communicate the user's intentions are required. Previous research has demonstrated that significant electrophysiological signals can be detected from muscles below the site of lesion despite the absence of visible muscle contractions. Classifying patterns of remaining surface electromyographic (SEMG) activity would increase the number of possible control signals, thus greatly enhancing the available information about user intention, potentially allowing patients with higher degrees of injury to use the assistive technologies. In this project, an interactive interface was designed using LabVIEW to collect and classify the activation of upper limb muscles, and evaluate the real-time classification accuracy achievable using different parameter combinations. Signals were collected on a non-disabled subject via a data acquisition system, filtered to remove noise, then amplified for ease of processing. Four time-domain features from the Hudgin's Approach were extracted from each muscle signal. Different muscles were found to display distinctively different SEMG patterns and features during different movements on the interactive interface in real-time. This finding suggests that it is possible to distinguish users' intention via the four time-domain features of the sEMG. A Linear Discriminant Analysis (LDA) classifier is currently being implemented as part of the interactive interface, with a goal of $70 \%$ accuracy classifying the movement the user intends to perform.

\section{Promoting Commercial Uses of Clinical Cardio- vascular Diseases Indication Wearable Devices}

\section{Haolin Liư', Daniel Franklin² \\ ${ }^{1}$ Engineering Science, University of Toronto \\ ${ }^{2}$ Institute of Biomedical Engineering, University of Toronto}

Multi-spectral photoplethysmography (PPG) is a method to measure health data by analyzing the lights with various wavelengths and penetrations that are absorbed or reflected in the blood vessels of living tissues. The multi-spectrum data shows the time delay in blood flow, which can be further analyzed for detections of early-stage heart failure and other cardiac problems. However, such application is only accessible for clinical use and limits its benefits for the general population. The main design objective of this research project is to enhance and commercialize the original medical device by improving usability, thus making it more beneficial for the stakeholders. Since the primary stakeholders are hospitalized elderlies with heart problems, due to their incomprehension of new technologies, the foremost requirement is ease-ofuse and accessibility, while ensuring the accuracy of the measurements. During the initial phase, I researched credible marketing surveys of customer preferences for wearables. By applying the engineering design strategies I have obtained from my study, my supervisor and I chose a ring- form for the device. Moreover, to avoid creating inconveniences in daily movements, I facilitated the team with circuit layout designs by creating digital and physical prototypes to optimize the size of the device. The next step of the project is to do bench tests on prototypes against approved medical devices to ensure accuracy. Today, the trend of wearables is tremendously increasing. We believe our device will bridge the medical and commercial aspects of the wearable market and has a great potential to benefit the general population.

\section{Maximizing Signal-to-Noise Ratio in DNA Gates for Non-Linear Disease Classification}

\author{
Jason J. Zhang ${ }^{1,2}$, Ryan C. Lee², Leo Y. T. Chou ${ }^{2}$ \\ ${ }^{1}$ Engineering Science, University of Toronto \\ ${ }^{2}$ Institute of Biomedical Engineering, University of Toronto
}

Gene expression profiling is a diagnostic method that can classify polygenic diseases like Parkinson's disease, diabetes, and cancer by analyzing gene regulation. A typical workflow involves collecting a patient's biological sample, transporting it to a central laboratory, extracting nucleic acids, and using microarray/sequencing technology with machine learning to predict disease. Researchers have simplified this workflow by designing molecular machine learning models that can profile gene expression and predict disease in vitro; however, these systems can only solve linear classification problems. We are designing a molecular multi-layer perceptron (MLP) that can solve non-linear classification problems in vitro, such as classifying a patient's disease as bacterial, viral, or 
noninfectious. The current focus of the project is optimizing the signal-to-noise ratio (SNR) of the MLP's functional unit, the node. The MLP's nodes are deoxyribonucleic acid (DNA) gates, partial duplexes of DNA that accept a complementary nucleic acid primer as an input. A strand- displacing polymerase then displaces the output strand from the duplex, allowing the output strand to activate a downstream gate. However, signal leakage is an issue with DNA gates due to impurities like truncation products and dehybridized oligonucleotides. We developed a protocol for removing impurities from circuit components using polyacrylamide gel electrophoresis, which has increased our nodes' SNR three-fold. We predict that a higher SNR will result in more accurate computations by the MLP and a higher prediction accuracy when classifying disease. Our next steps are to investigate how signal leakage impacts gates with multiple upstream and downstream interactions.

\section{Engineered Heart Valve Tissue for Preclinical Testing in a Pig Model}

Tina Sokhanvar ${ }^{1,2}$, Neda Latifi ${ }^{1,2}$, Nataly M. Siquei$\mathrm{ra}^{2}$, Shirley Chung ${ }^{2,3}$, J. Paul Santerre ${ }^{2,4}$, Craig A. Simmons $\mathbf{s}^{1,2,3}$

\section{${ }^{1}$ Department of Mechanical and Industrial Engineering, University of Toronto}

${ }^{2}$ Translational Biology and Engineering Program, Ted Rogers Centre for Heart Research

${ }^{3}$ Institute of and Biomedical Engineering, University of Toronto

${ }^{4}$ Faculty of Dentistry, University of Toronto

Congenital heart diseases are the most common type of birth defect. Often, these diseases involve dysfunctional heart valves, and require surgery for valve repair or replacement. However, current patches and prosthetic valves cannot grow and have limited lifespans, making them inadequate for pediatric patients. Heart valve tissue engineering aims to combine cells and biomaterials to create living replacement tissue for damaged valves. The challenge is to develop tissue whose composition and structure parallel that of the extracellular matrix (ECM) of native valves. As a first step towards clinical implementation, we are engineering heart valve tissue using pig umbilical cord perivascular cells (PUCPVCs) to be tested in pigs. In 2D culture, we observed that PUCPVCs produce significant amounts of collagen, elastin, and sulfated glycosaminoglycans (S-GAGs), major ECM components of native valves. Here, we grew PUCPVCs on a polycarbonate polyurethane scaffold with an aligned fibrous structure $(0.38 \pm 0.16 \mu \mathrm{m}$ diameter). We hypothesized that the scaffold would direct cell alignment and ECM biosynthesis, leading to 3D engineered tissue with properties mimicking native valves. We observed PUCPVCs aligned with scaffold fibers and had an average density of $30.4 \times 10^{\wedge} 6 \pm 7.75 \times 10^{\wedge} 5$ cells $/ \mathrm{cm}^{\wedge} 3$. Using biochemical assays, we measured $1.39 \pm 0.39$ $\mu \mathrm{g} / \mu \mathrm{g}$ DNA of collagen and $8.51 \pm 1.34 \mu \mathrm{g} / \mu \mathrm{g}$ DNA of S-GAGs. We further confirmed tissue development on both sides of the scaffold using scanning electron microscopy. Overall, these results demonstrate the feasibility of 3D culture protocols for designing tissue grafts for implantation in a porcine model for preclinical study.

\section{Novel insulin dependent sensor cell line to test viability of insulin variants}

\section{Jay Shah, Smarth Narula}

Institute of Biomaterials and Biomedical Engineering, University of Toronto

In Type 1 diabetes, insulin degraded through the proteasome is presented in the form of peptides onto the cell surface through the MHC1 MHC2 antigen presentation system. $T$ cells recognize these antigens and target the beta cell for destruction. Immunocloaking insulin peptides is an approach that entails replacing the native insulin gene with a variant to avoid immune recognition. Validating whether this new insulin variant is properly secreted and appropriately regulates gene expression is currently a process that requires multiple assays. The purpose of this project is to generate a sensor cell line that tests whether the variant insulin gene has been incorporated into the DNA, and whether the insulin is secreted and binds effectively to insulin receptors to elicit downstream effects. This cell line assesses these three checkpoints simultaneously and can evaluate thousands of variants in one assay. The cell line is designed by identifying genes that are upregulated by insulin binding and replacing them with green fluorescent protein. Gene targets have been identified through literature searches and are currently being validated through qPCRs, RNA sequencing, and western blots. Ideal targets will have at least a 7-fold relative increase in expression within 30 minutes of insulin presentation compared with basal expression levels in insulin-free 
conditions. The cell line will be co-cultured with beta cells expressing variants of insulin to identify compatible insulin variants in vitro. Rapidly assessing the viability of these variants will play a key role in identifying insulin variants that will be used to treat Type 1 diabetes.

\section{Single-cell RNA sequencing analysis of human adipose tissue towards the arterial endothe- lialization of small- diameter vascular prosthe- ses}

Kirtana Devaraj ${ }^{1}$, Jeremy Antonyshyn²,3, J Paul Santerre ${ }^{1,2,3,4}$

${ }^{1}$ Department of Chemical Engineering and Applied Chemistry, University of Toronto

2Institute of Biomedical Engineering, University of Toronto

${ }^{3}$ Translational Biology and Engineering Program, Ted Rogers Centre for Heart Research

${ }^{4}$ Faculty of Dentistry, University of Toronto

Introduction: Synthetic vascular prostheses exhibit poor long-term patency at internal diameters $<6 \mathrm{~mm} 1$. The endothelialization of these small-diameter vascular prostheses from short segments of explanted veins has been shown to improve their efficacy, but they are prone to accelerated atherosclerotic disease progression1. This is hypothesized to be a manifestation of grafting venous endothelium into the arterial hemodynamic environment1. The purpose of this investigation was to determine whether arterial endothelium can be harvested from human fat. Methods: Publicly available single-cell RNA sequencing data of human adipose tissue2 was analyzed using Seurat ( $R$ programming) and PANTHER (gene ontology). The stromal vascular fraction was first segmented on the basis of CD45, CD52 and CD31 expression. The endothelium was defined as the CD45- CD52-CD31+ population. The endothelium was further subclustered, and the identities of its subpopulations were determined using PANTHER. Potential cell-surface markers of the arterial subpopulation were determined using PANTHER. Results: Human fat was comprised of $20.63 \%$ CD45+ CD52+ leukocytes, 77.94\% CD45- CD52- CD31- adipose tissuederived stromal/stem cells, and 1.43\% CD45- CD52-CD31+ endothelial cells. Three distinct subpopulations of endothelial cells were identified, putatively comprising arterial (26.60\%), venous (55.05\%), and lymphatic (18.35\%) endothelium based on their unique biological processes. ITGB3 (CD61), CD3E,
CLSTN2 and MSLN were identified as candidate cell- surface markers, present exclusively in the arterial endothelium, for isolation from human fat. Conclusion: Human fat is a potential source of arterial endothelium for the endothelialization of small-diameter vascular prostheses. Future steps include verifying the cell-surface markers for arterial endothelium experimentally after cross-referencing them with the literature and our existing proteomics data.

\section{Optimizing the power efficiency of perovs- kite tandem solar cells with artificial neural network}

\section{Aoran Jiao1, Hu Quee Tan², Erik Birgersson² \\ 1 Division of Engineering Science, University of Toronto \\ 2 Department of Mechanical Engineering, National University of Singapore}

Solar photovoltaic energy is one of the fastest growing renewable energy sources according to Natural Resources Canada. Perovskite solar cell (PSC) is an increasingly popular photovoltaic device for its high efficiency, low cost and carbon footprint. Researchers have developed accurate optoelectronic models to simulate and evaluate the performance of PSC, but efficiency prediction is timeconsuming which hinders further optimization studies. To bridge this gap, the purpose of this research is to develop an artificial neural network (ANN) for efficiency prediction and to determine the optimal geometric parameters of a bifacial 4-terminal all-perovskite-tandem-solar- cell under different albedos. The experimental procedure includes data collection, ANN hyperparameter tuning, and global optimization. First, the developed optoelectronic model generates the geometric parameters with their corresponding efficiencies using different sampling techniques. These datasets are then used to train an ANN and determine the optimal hyperparameters using Bayesian optimization. With the trained ANN, the genetic algorithm is employed to find the geometric properties that yield the highest efficiency at different albedos. Preliminary results show that a shallow ANN with radial basis activation function and 38 hidden nodes consistently produces a low mean square error less than $10^{-4}$ with a computational time 3000-4000 times faster than the optoelectronic model. The genetic algorithm increases the average optimal efficiency to $32 \%$. More complicated models such as convolutional neural networks will be explored in future studies with effective 
visualization techniques including the parallel coordinate plots to better understand the optimization process. These results will guide the manufacturing of high-efficiency PSCs and will be applicable to further optimization studies in solar energy research.

\section{Examining the Potency of e-Fuels in Mitigating Climate Change through The Integration of e-Fuels into a Dynamic Fleet Model of the Uni- ted States Light Duty Vehicle Fleet}

Ikenna Okeke ${ }^{1}$, Alexa Barke², Riddhiman Roy ${ }^{3}$, Alexander Milovanoff ${ }^{1}$, Daniel Posen ${ }^{1}$, Heather Maclean ${ }^{1}$

\section{${ }^{1}$ FASE Civil Engineering \\ ${ }^{2}$ FASE Mechanical and Industrial Engineering \\ ${ }^{3}$ FASE Engineering Science}

As the world grapples with climate change, automotive manufacturers are looking to e-Fuels to help reduce emissions without requiring complete overhauls of existing infrastructure. To determine the climate impact of such fuels, models such as The Fleet Life Cycle Assessment and Material Flow Estimation model (FLAME), developed by Milovanoff and others, are used. FLAME projects the renewal of lightduty vehicles (LDVs) in the US to predict GHG emissions until 2050. E-fuels, defined by CONCAWE as gaseous or liquid fuels sourced from renewable electricity, are a new area of research. This project seeks to expand the FLAME to include e-Fuels into its pathways to investigate the propagation of these e-Fuels over their life cycles and their impacts on US GHG emission targets. This project will integrate suitable pathways into FLAME along with potential new technologies, expanding the model. Currently, a literature review is underway to examine e-fuels and their mitigation pathways. In addition, the FLAME model is being studied for potential insertion points and scoping. Following this, such pathways will be compared along depth, system boundary, data transparency and impacts, and implemented into FLAME. It is expected that e-Fuels will have a significant impact on the emissions of the US LDV fleet; however, it remains to be seen how that impact varies with deployment year, penetration and cost. Such results are of great interest to automotive manufacturers since these results could encourage the shift away from fossil-fuels, making e-Fuels a potent tool in the fight against climate change.

\section{Fundamental Investigation of Organic Electrode in Non- Aqueous Aluminum-ion Batteries}

\section{Matthew Desmarais ${ }^{1}$, Erica Wang ${ }^{1}$, Kok Long Ng², Gisele Azimi ${ }^{1}$ \\ 'Department of Chemical Engineering and Applied Chemist- ry \\ ${ }^{2}$ Department of Materials Science and Engineering}

For years, the energy sector has been dominated by the presence of rechargeable lithium-ion batteries. With its use spanning applications in devices such as phones, laptops, and even electric vehicles, its presence is vast and ever-growing. Although Li-ion batteries are vastly used and commercially available, what many have forgotten to consider is the long-term sustainability of such batteries. The problem is that the world is experiencing deficiencies in crucial Li-ion battery materials such as $\mathrm{Ni}, \mathrm{Co}$, and $\mathrm{Li}$. With the imminent exhaustion of Li-ion battery materials on the horizon, efforts have focused on replacements such as $\mathrm{Na}, \mathrm{K}, \mathrm{Ca}, \mathrm{Mg}$, and Alion batteries. Due to the aluminum's high natural abundance, low cost, and high gravimetric and volumetric capacities, the Al-ion battery has received considerable attention. The goal of this project is to explore the use of organic-based electrodes in rechargeable Al-ion batteries. Since 2015 , the vast majority of Al-ion batteries have been based primarily upon AlCl4intercalation and adsorption into carbonaceous cathodic materials such as graphene. Although this mechanism has been proven to work, research efforts have become tunnelvisioned upon this mechanism. In an effort to disrupt the Alion battery space and advance current research, our team is investigating a novel organic 5,7,12,14-Pentacenetetrone (PT) cathode. It is expected that this battery will enable the superior Al3+ intercalation mechanism, allowing Al-ion batteries to reach new heights in performance, bringing the world one step closer to a wide-scale commercialization of post lithium-ion batteries.

\section{Evaluating Carbon Capture and Utilization Pathways for Reducing Emissions of Canadian Methanol Production}

Clara Fleisig ${ }^{1}$, Adriana Gaona ${ }^{2,3}$, Heather L. MacLe$a^{4}$, Bradley A. Saville 2,3 


\section{${ }^{1}$ Engineering Science, University of Toronto}

2 Department of Chemical Engineering and Applied Chemistry, University of Toronto

${ }^{3}$ Laboratory of Bioprocess and Enzyme Technology

${ }^{4}$ Department of Civil and Mineral Engineering, University of Toronto

Methanol is an important chemical commodity used in a variety of commercial processes. To meet growing demand, Canada is developing plants that will potentially increase annual methanol production by $6.6 \mathrm{Mt}$. The conventional process for methanol production is disadvantaged by its high average greenhouse gas (GHG) intensity of $0.6 \mathrm{~kg} \mathrm{CO2-}$ eq / $\mathrm{kg}$ of methanol produced and dependence on natural gas. Thus, it is in Canada's interest to explore alternative pathways. Carbon capture and utilization for methanol production (MeOH-CCU) shows potential for GHG abatement. $\mathrm{MeOH}-\mathrm{CCU}$ pathways utilize $\mathrm{CO}$, which is otherwise emitted into the atmosphere. This study's objectives are to analyze the life cycle GHG impacts of methanol production via four $\mathrm{CCU}-\mathrm{MeOH}$ pathways and compare them with conventional pathways in a Canadian context. To the best of our knowledge, a comparison of these pathways has never been conducted in detail. Gate-to-gate and cradle-to-gate life cycle assessments are developed to evaluate and compare the GHG emissions of the pathways. Data regarding flow rates, resource extraction, processing and transport are obtained from literature and analyzed in MatLab. Preliminary results show that direct hydrogenation is the most promising pathway for production outside of Alberta, where electricity grids are less carbon intensive. However, electroreduction avoids challenges associated with hydrogen and natural gas feeds faced by other pathways. Future work involves simulating electroreduction to determine its potential when integrated with an ethylene-CCU process. This study aims to guide research and development by determining potential GHG emissions reductions for methanol production considering provincial conditions.

\section{Enhancing First Responders' Safety in Fire Inci- dents using an Interactive Hazardous-Material Detection Platform}

\author{
Yuchun Feng ${ }^{1}$, Zijian Wang ${ }^{2}$, Huifeng $\mathrm{Wu}^{3}$ \\ ${ }^{1}$ Engineering Science, University of Toronto
}

2 Department of Mechanical and Industrial Engineering, University of Toronto

${ }^{3}$ Department of Computer Science, University of Toronto

During a freight fire emergency, proper detection and respondence to unknown chemicals can help control the spread of a fire and prevent potential explosions. Failing to identify the hazardous materials in a timely manner might obstruct or even mislead the use of the correct fire extinguishers for the first responders, resulting in heavy casualties and economic losses. Thus, in fire incidents, it is crucial to shorten the decision-making time required to identify the emergency with an interactive hazardousmaterial detection platform, and that the overall damage could be minimized. In collaboration with the National Research Council of Canada (NRC), we are developing a multifunctional, robust interface as an aid in freight fire incidents. Functional requirements include showing the detected materials precisely and recommending the first responders with the corresponding emergency response guides and strategies. Non-functional requirements include that the platform should be user-friendly and easy-tounderstand for first responders who are not familiar with combustion processes. Our metrics unit revolves around our objectives including user satisfaction, usability, and efficiency. It will be measured by the average time (in seconds) taken by the user to navigate through the end-to-end process. With an understanding of our clients' needs, our procedure is to first confirm the most suitable software development tool, and then code the software and its features including part per million (PPM) visualization and integration of machine learning model ROCKET with time series data. In the near future, we will conduct testing with the NRC researchers to assess and improve the software upon their feedback. Taking account of these features, the software is believed to play an essential role for first responders at the fire scene once implemented.

\section{Design and Evaluation of a Water Treatment System for Medical Equipment Cleaning in Re- mote Hospitals}

\section{Dina Bernstein}

Department of Mechanical \& Industrial Engineering, University of Toronto 
Water is important for many applications, including cleaning and sterilization in hospital settings. One facet of water quality is hardness, which is caused by the presence of high levels of dissolved calcium and magnesium. Using such water to cleanmedical equipment in hospital settings can result in scaling and limit the effectiveness of sterilization procedures. Various global standards exist for water hardness for this application, but they often go unmet in developing communities. This project works with Haydom Lutheran Hospital in Tanzania, a hospital facing water hardness issues requiring them to regularly replace medical instruments. This work looks at potential water treatment technologies which could be integrated to reduce hardness in the equipment sterilization process. The solution needs to be cost-effective, require little additional action by hospital employees, and minimize water and energy usage. Through an analytical design process, Electronic Water Conditioning (EWC) was identified as a promising treatment method. EWC uses a coil-induced magnetic field to change the chemical structure of hard water ions which cause scaling. Advantages of this method include simplicity in implementation and use; however, it is not well-studied and has not been evaluated for medical instrument cleaning. Therefore, the next stage of the project will involve evaluating EWC, using experiments which replicate hospital conditions and Tanzanian water quality. Efficacy will be measured using water quality testing and scaling imaging. If successful, this method has the potential to be easily implemented into water circuits for remote hospitals, increasing medical equipment effectiveness and overall healthcare quality.

\section{Numerical Analysis of Macrotransport Pheno- mena in Concentrated Suspensions}

\section{Mingde Yin ${ }^{1}$, Arun Ramchandran² \\ ${ }^{1}$ Engineering Science, University of Toronto \\ ${ }^{2}$ Complex Fluids Laboratory, Department of Chemical Engi- neering, University of Toronto}

Macrotransport refers to the transfer of mass, energy, and other quantities over length scales much larger than molecular sizes. These processes are described most commonly at microscopic continuum length scales, a famous example being the Navier-Stokes equations, describing momentum transfer within Newtonian fluids. While computational fluid dynamics approaches can accurately model simple fluid materials, simulating particle-particle interactions in concentrated suspensions at macroscopic scales using microtransport models is hideously impractical for even modern computers. For instance, Stokesian dynamics approaches describe each particle in a suspension individually, but simulating every particle within a practically useful volume is computationally prohibitive. Macrotransport equations average their microscopic counterparts on larger length scales, creating computationally solvable problems. The "Coffee Ring Effect" describes a phenomenon where suspension particles tend to deposit around the perimeter of a droplet, as seen with coffee spills. In suspensions with strong surface tension effects, there are recirculating flows resulting in the creation of stagnation points within the suspension, disturbing the ring pattern. Presently, there exist no macrotransport models explaining particle migration phenomena around stagnation points. This work uses a macrotransport model in analyzing pressure-driven flow of a concentrated suspension of neutrally-buoyant, non-colloidal particles between two parallel plates. The model was applied to several stagnation point geometries implemented in COMSOL Multiphysics for comparison against experiments. Findings show qualitative similarity between simulation results and experiments in simple geometries, with new modifications implemented for more complicated scenarios. Further simulations in different geometries will motivate future experiments, and allow for improvements to the model.

\section{Improving the multifunctionality of polylactide (PLA) using graphene oxide nanoribbons (GONR) as nanoparticle fillers}

\section{Anthony Tuccitto', Chris Kwon², Saeed Habibpour ${ }^{3}$, Patrick Lee ${ }^{4}$ \\ Department of Mechanical and Industrial Engineering, University of Toronto}

${ }^{2}$ Engineering Science, University of Toronto

${ }^{3}$ Department of Chemical Engineering; University of Water100

${ }^{4}$ Department of Mechanical and Industrial Engineering, University of Toronto

With the concerns surrounding environmental pollution, replacements for petroleum-based polymers are sought for due to their poor recycling and degradation abilities. 
Polylactide (PLA) is a bio-derived, compostable polymer that could replace the said unsustainably sourced polymers, and also features competitive material costs and compostability compared to other biobased polymers. However, PLA suffers from insufficient stiffness and strength for large-scale industrial applications, as well as slow crystallization kinetics and poor thermal resistance. Recent research efforts have focused on using various nanoparticle fillers to enhance PLA's properties, including cellulose nanofibres, graphenes, and carbon nanotubes (CNT). However, such fillers tend to agglomerate when incorporated into PLA, reducing the filler-matrix contact surface area and hence the achievable improvements. Graphene oxide nanoribbon (GONR) produced from oxidative unzipping of CNT is a promising alternative to traditional filler materials given its high lengthto-width aspect ratio and its higher surface area, which could suppress stacking and agglomeration. Based on previous studies, there is a promising chance that GONR could enhance PLA's properties. Owing to the high surface-to-volume ratio, it is hypothesized that the incorporation of a very small concentration of GONR ( $<1$ wt\%) can overcome PLA's poor crystallization kinetics while also improving its stiffness and strength with minimal biodegradability compromises. In this work, we will explore mixing procedures to effectively incorporate GONR into PLA, demonstrate the scalability of the process, examine the fracture surface features using scanning electron microscope (SEM), evaluate mechanical performance using dynamic mechanical analysis (DMA) as well as crystallization kinetics using differential scanning calorimetry (DSC).

\section{Uniform Mixing of Steel Fibers in Piezoresistive Composites with a Static Magnetic Field}

\section{Melissa Ma1, Mohammadamin Jamshidi² \\ ${ }^{1}$ Department of Mechanical and Industrial Engineering,} University of Toronto

2 Department of Mechanical and Industrial Engineering, University of Toronto

Piezoresistive composites are used in wearable medical devices, self-sensing structural materials, and many more applications due to their electrical response to external stimuli such as stress, light, and temperature. Common techniques of mixing the conductive phase with the matrix are by hand- mixing or using electromechanical stirrers. However, dense conductive phases such as steel fibers tend to settle at the bottom of the mixture over time. The resulting non-uniform mixing could attenuate the composite's response signal to external stimuli. To address this gap, this project aims to develop a means to uniformly mix steel fibers via a static, spatially-varying magnetic field and quantitatively evaluate the efficacy of the proposed setup. The experimental setup consists of two steel magnets mounted above and below the curing sample at variable positions. We fabricate our composites by combining $0.45 \mathrm{~g}$ of $4 \mathrm{~mm}$ steel fibers and $50 \mathrm{~g}$ of DragonSkin ${ }^{\mathrm{TM}}$ silicone matrix. We use edge detection algorithms in MATLAB to segment individual fibers from microscope images of our sample, and then quantify the average fiber density throughout the cured sample. Preliminary results show that the proposed setup increases sample uniformity by $>40 \%$. Since microscope images are of limited magnification and resolution, the next step is to examine samples via a scanning electron microscope. Then, we will determine the optimal field location by iteratively adjusting the magnetic field profile. This research will improve the performance of piezoresistive composites in critical applications such as healthcare and infrastructure by providing a novel approach to ensure more uniform mixing in samples.

\section{A DC-DC Boost Converter for Powering Ultra- sonic Transducers in High-Speed Automotive Applications}

\section{Liza Zoubakina ${ }^{1}$, Wai Tung $\mathrm{Ng}^{2}$ \\ 1 Division of Engineering Science (ECE), University of Toronto \\ ${ }^{2}$ The Edward S. Rogers Sr. Department of Electrical \& Com- puter Engineering, University of Toronto}

Ultrasonic transducers transmit and receive sound pulses to detect nearby objects. The piezoelectric effect enables transducers to convert electrical energy to mechanical deformations and vice versa. Piezoelectric ultrasonic transducers have gained traction in short-range Driving Assistance Systems (DASs) such as car-parking assistance. However, recent challenges in high- speed blind spot detection arise due to the severe dampening of airborne sound. To improve sensor accuracy, transducers must be excited by higher-amplitude voltage pulses to transmit 
higher-amplitude waves. Our goal is to design a DC-DC switching power converter which steps up a typical $12 \mathrm{~V}$ vehicle battery supply to an $80 \mathrm{~V}, 0.1 \mathrm{~A}$ output suitable for powering transducers in high-speed automotive applications. Key design objectives of a DC-DC converter are to maximize the power conversion efficiency and power density over a range of loading conditions. Transducers require a regulated supply voltage, as evaluated by the transient settling time and load regulation metrics. To achieve these objectives, a closed-loop three-level boost converter topology was implemented. This multilevel structure exhibits higher power efficiency compared to conventional boost topologies. Gallium nitride (GaN) semiconductor devices are selected due to their lower switching and conduction losses compared with their silicon-based counterparts. Circuit simulations showed a peak power conversion efficiency of $91.4 \%$ at 0.1 $A$ and a $0.1 \%$ load regulation. A PCB (Printed Circuit Board) will be constructed to verify the performance of the proposed $1 \mathrm{MHz}$ boost converter. The proof-of-concept prototype will demonstrate the viability of GaN-based three-level boost topology in energizing ultrasonic transducers for automotive applications.

\section{Designing GaN-based DC-DC Buck Converter}

\section{Selena Liu', Wai Tung $\mathrm{Ng}^{2}$}

${ }^{1}$ Division of Engineering Science (ECE), University of Toronto

${ }^{2}$ The Edward S. Rogers Sr. Department of Electrical \& Computer Engineering, University of Toronto

Silicon semiconductor devices are used in nearly all power electronic systems including computers, home appliances, and power grids, but are reaching a limit in energy density. Gallium Nitride (GaN), a wide band-gap semiconductor, has the potential to increase the efficiency and reduce the size of these systems due to its favourable material properties including high electron mobility. The purpose of this research is to use GaN High-Electron-Mobility Transistors to design a power-efficient 12 to $5 \mathrm{~V}$ DC-DC buck converter to demonstrate the potential of GaN applications in low-power portable electronics. The key objective will be to maximize the power efficiency while accommodating for large and sudden changes of current draw that occur during power-on, sleep, and shut-off modes. Power efficiency is measured by the ratio of the output to input power. This metric is compared over a range of switching frequencies between $500 \mathrm{kHz}$ and $2 \mathrm{MHz}$ and current draw between $100 \mathrm{~mA}$ to $10 \mathrm{~A}$ to imitate practical applications in low-power electronics. Passive component values were simulated on PLECs and MATLAB to determine the stability and settling time of the open-loop system and an analog compensator is designed as a detachable module to boost the phase margin. The converter and compensator are fabricated and tested on a printed circuit board (PCB). We predict a $90-96 \%$ power efficiency, but future work will focus on reducing parasitic losses by optimizing the PCB layout. The impact of this work can lead to high performance electronics through the commercialization of GaN devices.

\section{Design Domain Pre-Processing for Efficient To- pology Optimization}

\section{Adele Crete-Laurence ${ }^{1}$, Craig Steeves $^{2}$ \\ 1 Engineering Science, University of Toronto \\ ${ }^{2}$ Advanced Aerospace Structures Lab, UTIAS}

Topology optimization is a mathematical structural design method used to find the optimal material layout in a specified design domain such that compliance is minimized for given loads and boundary conditions. In the Solid Isotropic Material with Penalization (SIMP) method, finite element analysis (FEA) is used, with the design variables being the element densities. This research aims to investigate, create, and test methods for design domain processing during early iterations that lower the computing power required to find an optimal structure. Ideal structures can be obtained through topology optimization without a preconceived configuration. The domain may contain thousands of elements, but a design that only contains material in a low percentage of those elements is often desired. There is, however, minimal discussion of removing elements during topology optimization in the literature. To preserve the method's accuracy, it is essential that the final design differs as little as possible from the result obtained with a full domain. Efficiently determining which elements to delete is a key aspect of the research. Metrics including density, sensitivity of the objective function, von Mises stress, and principal stress are examined, along with criteria varying from conservative to aggressive. The investigation, conducted in MATLAB, begins in two dimensions to gain an intuitive understanding before shifting to three dimensions, where a greater number of 
elements magnifies the benefits of altering the domain. Next steps include modifying the code for applications in additive manufacturing and remeshing the domain more finely after removing elements.

\section{Extracting transferable features from sensors for autonomous vehicle control}

\author{
Yewon Lee ${ }^{1}$, Julia Chae ${ }^{1}$, Pranit Chawla², Florian \\ Shkurti ${ }^{3,4,5}$ \\ ${ }^{1}$ Division of Engineering Science, University of Toronto \\ 2 Indian Institute of Technology, Kharagpur \\ ${ }^{3}$ Department of Computer Science, University of Toronto \\ ${ }^{4}$ University of Toronto Institute for Aerospace Studies \\ (UTIAS)
}

${ }^{5}$ Robotics Institute, University of Toronto

Autonomous vehicles rely on data like color images (RGB) and Light Detection and Ranging (LiDAR) to make steering decisions. Deep learning pipelines accurately predict steering commands when trained and tested on RGB and LiDAR images from the same simulation environment. However, they cannot be accurately scaled to real or unseen simulation environments unless re-trained from scratch, which is computationally expensive. To address this limitation, we propose splitting the pipeline into two training procedures: (i) training a model that extracts features from RGB and LiDAR pairs and then (ii) training a controller that linearly maps the extracted features to steering commands. We hypothesize that through part (i) our pipeline can learn features that are general enough that it can scale to new environments through only the secondary training procedure. To test this hypothesis, the feature extractor is trained, via contrastive learning minimizing the triplet margin loss, on a training dataset consisting of RGB and LiDAR pairs taken from three towns in the CARLA simulator. A controller is trained on data from each testing environment, consisting of different CARLA towns and the real-world. The trained models are used to drive an autonomous vehicle through $1 \mathrm{~km}$ of road in each environment. The cross track error is used to evaluate the models' performance. The baseline model, whose controller has been trained on the same environment as the feature extractor, achieved a cross track error of $2.5 \mathrm{~m}$. Achieving a similar magnitude of cross track error in other environments will demonstrate the generalizability of our method to new and real environments.

\section{The Effectiveness of High-Level Training for Ad- vanced Driver Assistance Systems (ADAS)}

\section{Ashna Jain, Chelsea DeGuzman}

Human Factors and Applied Statistics Lab, Industrial Engineering, University of Toronto

Many consumer vehicles now come with advanced driver assistance systems (ADAS), which can control vehicle speed and steering. While these systems can improve driver comfort and safety, several fatal collisions have occurred as a result of drivers over-relying on ADAS. Research has focused on reducing overreliance by training drivers on the system limitations found in an owner's manual. However, the literature indicates that this type of training may not be sufficient, especially as drivers tend to forget specific limitations over time. The purpose of the current study is to investigate whether a high-level approach to training is more effective than training on specific limitations. Two training videos were developed: a high- level video focusing on the overall fallibility of ADAS, and a baseline video that conveys limitations from an owner's manual. In this two-session virtual study, participants are shown one of the videos. Before and after watching the video, participants answer questions to assess their knowledge about how ADAS should be used. Participants return for a second session after one month to answer the same set of questions. Preliminary data analysis is currently in progress. It is hypothesized that participants who watch the high-level video will have a more accurate understanding of how the systems should be used, particularly in the second session as the baseline video is expected to result in lower knowledge retention. If the hypothesis is verified, this study would exemplify the need for high-level training strategies to improve consumer knowledge about ADAS and overall road safety.

\section{Computing Error-bounded Inverse Kinematics Solutions in Fixed- time using Low-Power Analog Circuits}

Pranshu Malik 
Edward S. Rogers Sr. Department of Electrical \& Computer Engineering, University of Toronto

A fundamental challenge for articulated robots is inverse kinematics (IK), which converts end- effector goals in theworkspace into viable joint configurations. In general, IK is a hard nonlinear problem for serial kinematic chains. Therefore, efficient methods that are applicable to a broad range of manipulators are important. Current IK methods (analytical or numerical) do not have a bound on the time to compute solutions and are not guaranteed to yield locally smooth solutions between two adjacent configurations, which may cause instability if iterative IK solutions are required. We propose an unconventional computation model with a digitally reconfigurable analog circuit to compute IK solutions in two stages. First, we find an approximate solution, within an error-bound decided by a frequency-scaling factor, by continuously evaluating the forward kinematics (FK) through a reconfigurable, time-varying matrix whose entries are combinations of sinusoidal signals. These signals are phaselocked and frequency-scaled sines of successive joint angles that help capture the non-linearity of FK naturally by varying over time. Next, another analog circuit block continuously refines the approximate solution using Jacobian-based methods to drive the solution closer to the goal. The model is parallelizable which enables the circuit to be used inside a high-rate control loop and also allows starting the search from any configuration. We demonstrate a proof of concept; a future fabricated circuit can be compared against the algorithmic methods for accuracy, throughput in solutions per second, and energy utilized.

\section{First-year Undergraduate Student Beliefs About Teamwork: A Qualitative Analysis}

\section{Inho Kim 1,2,3, Patricia Sheridan ${ }^{2,3}$}

${ }^{1}$ Division of Engineering Science, University of Toronto

${ }^{2}$ Troost Institute for Leadership Education in Engineering

${ }^{3}$ Institute for Studies in Transdisciplinary Engineering Education and Practice

Undergraduate student beliefs around Engineering Teamwork have been described in literature as unsophisticated, naïve, and even misdirected given the Nature of Engineering such as by Karataş et al. (2016). Furthermore, Karataş et al. state that regarding teamwork as a negative practice that lowers grades may stem from students' pre-university experiences. Research led by Missingham and Matthews (2014) also reveals that students view the collaborative process as "intrusive and confronting." Beliefs like these can negatively affect students' engagement in teamwork and the strategies they choose to work effectively. Thus, there is a need for Engineering Education to actively support students in their teamwork learning. This study investigates how the University of Toronto firstyear Engineering students' beliefs around Engineering Teamwork compare to those discussed in literature. Survey data was collected from first-year students in Winter 2014 to surface their prior teamworking experiences, and their beliefs around effective teamwork. Using qualitative analysis, these descriptive textual responses were used to identify themes that represented specific beliefs. These themes were then compared to the literature to determine if they were consistent. Themes regarding teamwork as inefficient due to experiences around unequal divisions of work and perceived skill disparity emerged as possible trends that reflect the literature. Through this research, the curriculum around first-year engineering design can be improved to respond to incoming students' beliefs about teamwork. Designing curriculum to better support students' understanding of Engineering teamwork and fill in students' knowledge gaps around the Nature of Engineering will enable greater student success in the future.

\section{Transdisciplinarity in Society and Engineering Education: A Literature Review}

Helen Tran, Gamen Liu

\section{Chemical Engineering, University of Toronto}

The concept of transdisciplinarity originated in the 1970s, and has since continued to develop in regard to its definition, scope, and applications. It emerged in response to the limitations of siloed disciplinary approaches in producing knowledge and solving wicked problems, a term that references global issues such as climate change and health disparities. Thus, transdisciplinarity was coined to describe the transcendence of knowledge between, across, and beyond disciplinary boundaries with unique viewpoints from science, policy studies, humanities, and economy. As transdisciplinarity initiatives have increased in the field of 
engineering education over the past few years, knowledge is lacking with respect to what "transdisciplinary engineering education" means and how it can materialize in practice. In this context, the purpose of this presentation is to share the work in progress of a literature review to trace the history of transdisciplinarity and its definitions, and identify approaches to potentially analyzing its current and future role in education, engineering, and society. For this purpose, we sought out literature using abstract and keyword search in engineering and education databases such as Compendex and ERIC. Our review recognizes two main approaches to transdisciplinarity: Nicolescuian and Zurich. However, the character of transdisciplinarity remains varied and broad as these approaches address different concerns and in nature, are methodological and phenomenological, respectively. Our results also show that the current scene of disciplinary education presents resistance to the unfamiliarity of transdisciplinarity. Moving forward, our research will contribute to situating ISTEP's intellectual worth in broader fields.

\section{Understanding the motivational barriers affec- ting students within first-year design teams}

\section{Osarugue Ize-lyamu1, Victoria Kerr²,3, Emily Moo- $\mathrm{re}^{3,4}$}

${ }^{1}$ Department of Mechanical and Industrial Engineering

2 Department of Chemistry and Chemical Engineering

${ }^{3}$ Institute for Studies in Transdisciplinary Engineering Education \& Practice

${ }^{4}$ Troost Institute for Leadership Education in Engineering

First year design courses use team based projects to foster communication and other industry- recognised social skills amongst engineering students. In first-year teams, student engagement is crucial for team effectiveness and success. Engineering education research has been conducted to better understand and improve the effectiveness of these teams as a whole. However, more research is needed to provide insight on the individual experiences of students within these teams. This is needed to understand and improve student engagement for those who are encountering team engagement difficulties. The purpose of this research study is to explore and identify the prominent motivational barriers that disrupts student engagement in first-year design teams. During the period of study, a phenomenographic methodology was employed. Informal student and instructor interviews were conducted and thematic qualitative analysis was used to better understand the prevalent motivational barriers affecting students in first year. This research will be used in preparation for a more detailed survey or interview instrument to be developed. In identifying the prevalent motivational barriers students face, this study will inform future research and will allow for increased empathy in instruction and curriculum development.

\section{Challenges of Collaboration with Computer Ai- ded Design (CAD)}

Jasmine Zhang ${ }^{1}$, Michal Davis ${ }^{1}$, Alison Olechows$\mathrm{ki}^{2}$, Shurui Zhou ${ }^{3}$

${ }^{1}$ Engineering Science, University of Toronto;

${ }^{2}$ Department of Mechanical and Industrial Engineering, University of Toronto;

\section{${ }^{3}$ Electrical and Computer Engineering, University of Toronto}

Collaboration with Computer Aided Design (CAD) plays a central role in hardware design processes. However, there remain many challenges of collaboration with CAD and limited classifications of these challenges in literature. We resolve this lack of understanding of collaboration with CAD challenges and potential solutions. Through literature review and qualitative interviews with CAD professionals, we formulate pain points, their underlying causes, and current mitigation strategies in collaborative workflows involving CAD. Several pain points and causes are not found in the literature review, possibly representing previously unknown areas. In total, we identify thirteen pain points and nineteen underlying causes. Six identified mitigation strategies currently in use resolve a subset of these pain points and causes but introduce eighteen of their own challenges. Future work in CAD development can use this classification of challenges to target the most critical areas for improvement.

\section{Bio-Traveller: Using Roblox to create an online, game-based learning environment for students to learn biological concepts}

\author{
Ally Cheung ${ }^{1}$, Foram Naik², Suhail Anuarr, Bianca \\ Ortiz ${ }^{4}$, Chuntat Lui ${ }^{4}$, Keith Pardee ${ }^{5}$, Jennifer
}




\section{Doucet $^{5}$, Reza Mohamadi ${ }^{5}$}

${ }^{1}$ Engineering Science, University of Toronto

2 Medical Sciences, Western University

${ }^{3}$ Department of Mechanical \& Industrial Engineering, University of Toronto

${ }^{4}$ Life Sciences, University of Toronto

${ }^{5}$ Faculty of Pharmacy, University of Toronto

Non-traditional learning methods have shown potential in supporting students' creativity, critical thinking, collaboration, and communication. With an increased need for remote learning during the COVID-19 lockdown, virtual gamebased learning offers an approach to engage students in challenging educational content while having fun. Pioneering projects, such as Fold.It and Phylo, introduced the concept of gamifying biology; however, there was a lack of engagement in the process of learning. We are currently developing a game named Bio-Traveller, powered by the Roblox game engine. It is constructed based on the Immersive VR learning model, a framework that is proven to promote user engagement during science learning. The model aims to draw players' focus, participation, and persistence within a task. Bio-Traveller immerses the players in a fictional world where they are the surviving members of an apocalypse. To restore science and technology, they time-travel to the labs of different famous scientists in human history and learn about their research. Our prototype experimented with diverse game styles, such as escape room, obstacle course, lab simulation, simon-says, puzzle, and storytelling. As well as covering "Biodiversity" (Grade6), "Cells" (Grade8), "Tissues, Organs, and Systems of Living Things" (Grade 9), and "Chemical Reactions" (Grade 10) from the Ontario Curriculum of Science and Technology. Future work will be devoted to studying users' experiences and measuring educators' attitudes towards using BioTraveller as a tool to teach science.

\section{Floating Point Computing With Coarse- Grained Reconfigurable Arrays}

\section{Adham Ragab¹, Omar Ragheb², Jason Anderson²,} Tianyi $\mathrm{Yu}^{2}$

${ }^{1}$ Division of Engineering Science, University of Toronto

${ }^{2}$ Edward S. Rogers Sr. Department of ECE, University of Toronto programmable hardware platforms that can be configured to implement application-specific accelerators to raise computational performance and energy efficiency vs. using standard microprocessors. CGRA-ME (CGRA modelling and exploration) is an open-source software framework under active development at UofT that permits the modelling, development and evaluation of new CGRA architectures. Presently, CGRA- ME is limited to CGRAs that can perform integer and logical operations. While integer/logical suffices for many applications, others, such as machine learning training, require floating-point computations. The goal of the research is to enhance the capabilities of CGRA-ME to include floating point. This involves 1 ) surveying existing open-source floating point hardware core libraries and assessing their area and performance, 2) integrating a selected library into CGRA-ME, and 3) the research, design, development, and test of a CGRA that leverages the new floating-point cores.

\section{Evaluating Current Knowledge Organization Strategies on Stack Overflow to Develop an Au- tomated Wiki Post Generator}

Arjun Sridharkumar¹, Hamza Dugmag², Shurui Zhou $^{3}$, Iftekhar Ahmed ${ }^{4}$

\section{Department of Computing Science, University of Alberta}

2 Engineering Science, University of Toronto

${ }^{3}$ Department of Electrical \& Computer Engineering, University of Toronto

${ }^{4}$ Department of Informatics, University of California Irvine

StackOverflow is the largest community-based, questionand-answering website used by programmers. Unfortunately, as documented in the literature as "unknown-item searches," this format creates an overwhelming number of posts that impede the retrieval of desired knowledge. StackOverflow alleviates this through Wiki Posts, where users can collaborate to collect links to the most useful posts into a single Wiki Post. However, compared to StackOverflow's database of over 21 million questions, the current Wiki Posts have collected around 5000 questions in total, indicating that the site remains unorganized. This project aims to extract data on the popularity and structure of current Wiki Posts to cluster using HDBSCAN because we have no insight into the different classes of posts. Then, using stratified sampling, we can identify the contents of effective Wiki Posts and the 
pain points associated with organizing knowledge. Moreover, we will detect type III code clones between Wiki Posts and their Official Documentation counterparts to outline the advantages of one over the other. Backed by qualitative analysis, we expect there to be shared features among highquality Wiki Posts, - such as a high number of external links - some of which mimic documentation structures. These results allow us to better understand how we can design Wiki Posts to boost collaboration, education, and innovation among developers without worrying about spending too much time searching for hidden knowledge. From this, we plan to evaluate various NLP models to propose an automated tooling method that generates Wiki Posts more efficiently to improve knowledge organization on StackOverflow.

\section{Engineering Students' Academic Engagement and Learning Motivation in the Online Learning Environment During the Pandemic}

\section{Qin Liu', Jenifer Hossain², Khaza Abdur Rouf², Jiyoun Lim $^{3}$}

${ }^{1}$ Institute for Studies in Transdisciplinary Engineering Education \& Practice, University of Toronto

2 Computer Engineering, University of Toronto

${ }^{3}$ Chemical Engineering, University of Toronto

Before COVID-19, engineering education mainly relied on in-person delivery. The outbreak of COVID-19 introduced an exclusive online instructional mode to engineering education, which dramatically reshaped students' abilities to engage with their courses. However, many engineering instructors lack experience in delivering courses online, and have limited understandings about how students' engagement with online teaching strategies affected their motivation for learning. Our study aims to examine the relationship between engineering students' academic engagement, including how students perceived teaching techniques and adjusted their learning strategies when learning online, and their learning motivation in the online exclusive environment during the pandemic. For this purpose, we analyzed data collected in May 2021 from an extensive survey about undergraduate engineering students' overall online learning experiences during the pandemic. Mixed methods are used for data analysis. Quantitatively, correlation analysis is conducted to inspect a relationship between students' academic engagement and their motivation. Qualitatively, thematic analysis is conducted to identify major themes in student comments in terms of environmental and individual factors affecting student motivation. Results show environmental factors improved accessibility of academic resources and flexibility in their time management, which positively impacted students' motivation. Our analysis also reveals that students demonstrated their agency by adjusting their learning strategies to effectively navigate the online learning environment. Our study suggests integrating these key factors to redefine future teaching strategies could help maintain students' learning motivation and engagement as engineering education is possibly shifting to a hybrid mode of delivery in the wake of the pandemic.

\section{Artificial intelligence for the analysis of ther- mally sprayed functional coatings}

\section{Parth Mahendru', Moussa Tembely², Prof. Ali Dolatabadi ${ }^{3}$ \\ 1 Engineering Science, University of Toronto \\ ${ }^{2}$ Concordia University, Multiphase Flow and Thermal Spray Laboratory \\ ${ }^{3}$ University of Toronto Centre for Advanced Coating Techno- logies}

The purpose of this paper is to utilize artificial intelligence in the forms of machine intelligence and computer vision to analyze Suspension Plasma Spray Coatings (SPS) and make predictions about its properties. SPS coatings are a recent development in the field of coating sciences and have applications in the aerospace industry as they can provide very thin, even nano-structured coatings, desirable for thermal applications. The surface performance of an SPS coating is dependent on numerous variables and process parameters that are expensive to physically experiment. Thus, using machine intelligence to predict these surface properties would circumvent the need for the aforementioned experimentation, save on time while offering added flexibility in designing a specific coating. The most information about a particular coating is given by the Sliding Angle, a measure of mobility of a fluid on the surface. We used a dataset from Sharifi (2017) consisting of SPS conditions and sliding angle to train models in regression, gradient boosting and neural networks to predict the sliding angle given initial 
test conditions. We concluded that the XGBoost performed the best for our dataset. Additionally, we developed an algorithm to match the predicted sliding angle to the actual images of the microstructures. Using which, we coded a Generative Adversarial Network to generate images of the microstructures corresponding to the predicted sliding angle. More experiments in the future mean more data, which would enable more accurate predictions. Regardless, current results show promise and can be used as a preliminary indicator of microstructure properties.

\section{Machine Learning Applications to Sports Injury: A Review and Proposal}

\section{Hanna Sigurdson ${ }^{1}$, Jonathan Chan² \\ ${ }^{1}$ Engineering Science, University of Toronto \\ ${ }^{2}$ School of Information Technology, King Mongkut's Univer- sity of Technology Thonburi}

As sports injuries increase in frequency in adolescents, and injuries in professional athletes create a detrimental impact on the sports industry, research surrounding preventing sports injuries becomes more prevalent. The mechanism for sports injury is well defined and includes intrinsic risk factors (age, psychology etc.), extrinsic risk factors (weather, training load etc.), and the inciting event. With the rise of machine learning $(\mathrm{ML})$, a variety of $\mathrm{ML}$ techniques have been applied to various sports injury aspects. The purpose of this work is to assess the current applications of ML to sports injury and identify areas of growth by a systematic analysis of applications to each injury element: intrinsic factors, extrinsic factors, and the inciting event. The literature review follows exclusion and inclusion criteria, and occurs on the databases Google Scholar, PubMed, and Scopus. Current underdeveloped areas are identified as: psychological effect, use of extrinsic factors, analysis of the inciting event, and application of the action recognition ability of videos and wearable technology. A proposed model responding to undeveloped areas uses computer vision to identify and eventually predict injuries based on video data of the inciting event. This concept leverages the non-intrusive availability of video, and aids in understanding and preventing injury. Further technical application of this proposition and these undeveloped areas should be undergone to expand on and improve sports injury prevention technology.

\section{Exploring Learning-Based Solutions to the Inver- se Kinematics Problem}

\section{Petra Alexson ${ }^{1}$, Filip Marić2,3, Matthew Giamou³,} Oliver Limoyo ${ }^{3}$, Jonathan Kelly ${ }^{3}$

${ }^{1}$ Engineering Science, University of Toronto

${ }^{2}$ LAMOR, University of Zagreb

${ }^{3}$ University of Toronto Institute for Aerospace Studies

The inverse kinematics (IK) problem is concerned with finding a set of possible joint angles given a desired position and orientation for the end-effector of a robotic arm. IK cannot always be solved with an analytical solution, and it becomes even more challenging when considering robots with complex structures and changing environments. Learningbased approaches have the potential to address some of these challenges, but numerical solvers currently offer better precision. This project evaluates an existing learning-based IK solution, distal teacher learning (DT), and investigates how using concepts from DT may improve the performance of a novel approach, generative graphical IK (GGIK). DT trains a neural network tailor-made to a specific robot's IK, relying on its kinematic parameters to minimize error between the input pose and the pose calculated from the predicted joint angles. GGIK leverages the graph-like structure of robots to create models using graph neural networks (GNNs), which accept inputs of variable size. Experiments on various robotic arms show that DT can achieve similar computation times to state-of-the-art numerical solvers, but it is less precise. GGIK has higher computation times and even lower precision than DT. Crucially, however, it can approximate IK solutions for arbitrary robots. One possible direction to build upon GGIK, investigated in this project, is to adapt DT to work with GNNs that take in simpler graph-based robot representations. GGIK has the potential to be part of an end-to-end learning-based motion planning system for arbitrary robots, especially if its performance gaps with other methods are addressed.

\section{Optimizing Consumer-Grade EEG Devices for Identification of Neurodegenerative Behaviour}

Sophie Sun ${ }^{1,2}$, Alberto Leone ${ }^{2}$, Phedias Diamandis $2,3,4,5$

${ }^{1}$ Engineering Science, University of Toronto 
${ }^{2}$ Princess Margaret Cancer Centre, University Health Network

${ }^{3}$ Department of Laboratory Medicine and Pathobiology, University of Toronto

${ }^{4}$ Laboratory Medicine Program, University Health Network

${ }^{5}$ Department of Medical Biophysics, University of Toronto

The increased use of personal biotracking devices has generated interest in the application of electroencephalogram (EEG) devices to non-invasively monitor brain activity. Where traditional EEG headsets can cost over $\$ 100000$, commercial and portable EEG devices are accessible for as little as $\$ 200$, offering the ability for population-level real time monitoring. However, the potential for these devices to detect various physiological and pathological states still needs to be optimized. To address this, I hypothesize that utilizing Al will enable portable EEG devices to detect meaningful electrophysiological differences. I aim to collect brain data using portable EEG devices (AIM1), and classify neurological states using functional imaging data (AIM2). Towards AIM1, I helped develop an online platform, Sibley, that independently guides subjects through various neurocognitive tests while capturing their EEG data using the 14 electrodes EPOC X EEG device $(\$ 800)$. By recording EEG data from 15 healthy subjects, I validated that this platform and the tests were significantly sensitive ( $p$-value $=0.0037$ ). Towards AIM2, I retrieved functional neuroimaging data of patients with autism spectrum disorder and control subjects to develop a machine learning workflow that classifies patients based on their brain connective maps. Specifically, I deployed a random forest classifier and achieved a stateof-the-art classification accuracy of 0.71 (area under the receiver operator curve $=0.75$ ). Through this project, I have developed a computational platform to collect and analyze functional neuroimaging data. Future work aims to integrate these components to create a fully automated pipeline for the detection of neuropathological disorders.

\section{Building Energy Consumption Forecasting: A Comparison of Gradient Boosting Models}

Abnash Bassi ${ }^{1}$, Anika Shenoy ${ }^{1}$, Arjun Sharma ${ }^{1}$, Hanna Sigurdson ${ }^{1}$, Connor Glossop ${ }^{1}$, Jonathan H. Chan ${ }^{2}$

\footnotetext{
${ }^{1}$ Engineering Science, University of Toronto
}

${ }^{2}$ School of Information Technology, King Mongkut's University of Technology Thonburi

Building energy consumption forecasting is essential for improving the sustainability of buildings in the context of addressing climate change. Accurate building load predictions are useful for energy efficient building design selection and demand-side management initiatives. Using historical building energy consumption data has allowed researchers to develop machine learning models to improve the accuracy of such predictions, beyond inefficient traditional approaches otherwise used by the building sector. This work examines gradient boosting machine learning models, namely LightGBM, CatBoost, and XGBoost, for the purpose of comparing their performance on a select dataset. These gradient boosting models are popular in Kaggle machine learning contest solutions but have not been compared formally for the application of building energy consumption predictions. This work applies the three gradient boosting algorithms to a synthesized dataset for a large office building in Chicago. Preliminary results from the presented comparison demonstrate that XGBoost outperforms both LightGBM and CatBoost as indicated by its higher R2 value and lower reduced normal RMS log error when trained on the selected dataset. These results seem consistent with previous literature indicating the success of XGBoost models in predicting building energy consumption and provide grounds for future testing and use of the model on larger, more diverse datasets.

\section{Extending the Capabilities for Radar Cross Sec- tion Calculations using Mie Series in Computa- tional Electromagnetics}

\section{Iliya Shofman, Damian Marek, Shashwat Sharma,} Piero Triverio

The Edward S. Rogers Sr. Department of ECE, University of Toronto

The design of complex electromagnetic devices, such as metamaterials and high-frequency computer processors, requires advanced Computational Electromagnetic (CE) solvers which obtain numerical solutions by solving Maxwell's equations. The acceleration and improvement of these time-consuming algorithms is an area of significant ongoing research. To evaluate the accuracy of new CE solvers during 
the research and development phase, the solvers' numerical results are compared to analytical results for simple cases, such as electromagnetic scattering by spheres. Analytical solutions to this problem can be calculated using the Mie series; the aggregate result of this calculation is known as the radar cross section (RCS). While several open-source libraries for Mie series calculations exist, those codes suffer from a lack of robustness for highly conductive objects. A literature review revealed that several existing libraries were unable to yield results for lossy dielectrics and at high frequencies - a significant problem considering the importance of these regimes for a wide variety of electromagnetic engineering problems. This research project identified and addressed the computational issues within one open-source library, enabling calculations for a broader range of frequencies and extremely lossy dielectrics while decreasing run-time and increasing code robustness. Amendments to the code were verified for mathematical correctness and physical reasonableness. The results of the improved code were in close agreement with several existing CE solvers and results in the literature, thereby establishing accuracy. As an outcome, the improved code has been made publicly available for broader use by scientific communities to enable research, electromagnetic design, and educational endeavors.

\section{Assessing the performance of viscosity measu- rement technologies}

\section{Daniel Foster ${ }^{1}$, Daniel MacGregor², Philippe La- voie $^{2}$ \\ ${ }^{1}$ Engineering Science, University of Toronto \\ ${ }^{2}$ University of Toronto Institute for Aerospace Studies}

A falling-ball viscometer is a device that calculates the viscosity of a fluid by timing the descent of a ball between two reference points. This can be accomplished using a stopwatch, but introduces significant uncertainties due to human error. It is desirable to use a measurement method that lowers viscosity-associated uncertainties in experimental fluid dynamics. This research sought to develop and compare a computer vision system and photogate system to minimize the uncertainty associated with oil viscosity measurements. The design objectives consisted of minimizing temporal uncertainty, variance of results, and sensitivity to experimental setup. The associated metrics were: temporal uncertainty in minutes, variance in minutes squared, and a qualitative assessment of robustness. A computer vision tool was developed to track the descent of a ball in a viscometer. Uncertainty was decreased through the use of a leastsquares linear fit to a plot of the ball's position, variance was reduced by using an ensemble of derived quantities, and sensitivity was decreased with a colour calibration system. A photogate apparatus was also created to measure the times at which the ball passes through two reference points on the viscometer. Uncertainty was decreased by using photogates with high temporal resolution, variance was reduced by using an ensemble of derived quantities, and sensitivity was decreased with the use of infrared emitters and receivers as the photogate apparatus. When evaluated against the defined metrics, the photogate system was superior to the computer vision tool, suggesting that it presents a favourable method for viscosity measurement.

\section{Automation of the FCET Wind Tunnel Smoke Flow Visualization Rig}

\section{Daniel Asad1', Suraj Bansal², Philippe Lavoie² \\ 1 Engineering Science, University of Toronto \\ 2 University of Toronto Institute for Aerospace Studies}

When analyzing the properties and features of flow around aerodynamic devices, there are quantitative techniques such as particle image velocimetry (PIV) and hot-wire anemometry, while the SFV method provides a simpler set-up and qualitative look at the flow field. The SFV technique consists of a nichrome wire that is coated in propylene glycol and is heated to create lines of smoke through the wind tunnel. It allows for the visual identification of key features such as boundary layers, laminar or turbulent flow and vortex shedding. Currently, each instrument in the setup is controlled manually and is only effective for flow speeds under $5 \mathrm{~m} / \mathrm{s}$, therefore limiting the precision of equipment usage for unsteady conditions and the range of possible cases to study. This project aims to increase the efficiency, precision, and versatility of the SFV setup by automating the components using a microcontroller, as well as implementing capacitors to rapidly discharge high levels of current for usage above $5 \mathrm{~m} / \mathrm{s}$. The desired circuit and code design will obtain inputs either from an encoder signal or a manual trigger and output precise, timed controls to the wire heating, the dispensing of propylene glycol, the camera, 
and the capacitors. Design solutions will be evaluated based on the metrics of usability, compatibility and responsiveness. Ultimately, this system maximized usage of SFV and aided in the studies of laminar separation bubbles. Through iterative design and evaluation of metrics, the created design was able to produce informative images in a consistent manner while additions are being made to augment the usage of the instruments through the ability to read and save data.

\section{Therapeutic Potential of Targeting Tumor-As- sociated Macrophages to Treat Therapy-Resis- tant Pancreatic Ductal Adenocarcinoma}

Michelle Nurse ${ }^{1,2}$, Ziting (Judy) Xia ${ }^{2,3}$, Ileana $\mathrm{Co}^{3,4}$, Alison McGuigan ${ }^{3,4}$

${ }^{1}$ Department of Cell and Systems Biology, University of Toronto

2 Department of Ecology and Evolutionary Biology, University of Toronto

${ }^{3}$ Department of Chemical Engineering and Applied Chemistry, University of Toronto

${ }^{4}$ Institute of Biomaterials and Biomedical Engineering, University of Toronto

Pancreatic ductal adenocarcinoma (PDAC) is one of the most aggressive forms of cancer, with a 5- year relative survival rate of $5-10 \%$. Contributing to its poor prognosis is the immunosuppressive nature of the tumor microenvironment (TME) and resistance to current therapies. Moreover, solid tumors such as PDAC often have a hypoxic TME which alters cell metabolism, further promoting tumor progression. The TME consists of secreted factors and several immune cells. In particular, tumor-associated macrophages (TAMs) are known to have a pro-tumoral role, downregulating the body's immune response and interacting with PDAC cells to promote tumor growth and metastasis. Hypoxia exacerbates these TAM-associated effects. Overall, TAMs not only contribute to PDAC progression but also directly oppose the action of treatments like chemotherapy and immunotherapy, which focus on enhancing cytotoxicity against tumor cells. This review summarizes the interactions between PDAC cells and TAMs in both normoxic and hypoxic conditions, focusing on their relation to cancer progression and therapy resistance. A search of recent literature from databases such as Google Scholar, PubMed and ScienceDirect was performed. The data was used to developed a schematic representation of
TAM-PDAC interactions, highlighting potential targets to treat PDAC. A CellPhoneDB analysis of interactions in PDAC patient samples was conducted to evaluate the relevance and frequency of hypoxia-specific interactions. Developing a better understanding of TAM-tumor interactions is critical to understanding the complexity of the TME. Based on our presented rationale, future therapeutic development for PDAC should seek to modulate the immunosuppressive TME, with TAMs as an important target.

\section{Computational Modelling of Angiogenic Factor Secretion In A Fibroblast-Endothelial Microflui- dics Platform}

\section{Jasnoor Guliani', Sina Kheiri², Noosheen Walji2,3, Edmond W.K. Young 2,3 \\ ${ }^{1}$ Department of Electrical and Computer Engineering, Uni- versity of Toronto \\ 2 Department of Mechanical and Industrial Engineering, University of Toronto \\ ${ }^{3}$ Institute of Biomedical Engineering, University of Toronto}

Angiogenesis, which is the development of new blood vessels from existing vasculature, is a key process in both normal development and metastasis of cancer cells. In vitro models are necessary for investigating the mechanisms of angiogenesis and developing anti-angiogenic therapies to prevent tumour growth. Microfluidic angiogenesis platforms enable researchers to carefully control microenvironments and recapitulate 3D tissue structures. To model the angiogenesis process, microfluidic platforms often include endothelial cells and a fibroblast component. However, the effects of spatial organization of fibroblasts on resulting angiogenic behaviour and factor secretion remain unclear. Mathematical and computational modelling enables rapid and simultaneous screening of various chemical and physical features in microfluidics platforms. Here we conduct a comparative numerical study of angiogenic sprouting on a microfluidic chip induced by fibroblasts in 2D monolayer, 3D dispersed, and 3D spheroid culture configurations. We study the diffusion gradients of select secreted factors (bFGF, VEGF) across a cell co-culture microfluidic chip and correlate computational results with measurements of vessel morphology, sprout distribution, and secreted factors taken from microfluidic models of each configuration. Our results show that secreted factor gradients vary in shape and 
quantity across the porous medium in response to fibroblast organization. We anticipate this work sheds light on how angiogenic sprouting dynamics are mediated by fibroblast configuration and enables purposeful selection of fibroblast organization in the microfluidic cell culture design process. Further, we hope to expand this work into a user-friendly standalone application that enables selective adjustment of device parameters for those with minimal modelling experience.

\section{Neural traffic in peripheral nerves: Extracting what matters}

\section{Katie Khodawandi ${ }^{1}$, José Zariffa²}

${ }^{1}$ Electrical \& Computer Engineering, University of Toronto

${ }^{2}$ Institute of Biomedical Engineering, University of Toronto

Peripheral nerves act as multi-lane highways that transport a mix of sensory and motor information in multiple directions. The ability to selectively stimulate and discriminate between neural pathways within peripheral nerves has clinically relevant applications in neurorehabilitation, including control of prosthetics and restoration of movement in paralyzed limbs using electrical stimulation. To this end, peripheral nerve interfaces (PNIs) are needed to record and/or stimulate neural activity in peripheral nerves. However, there exists a trade-off between invasiveness and recording selectivity. Minimally invasive PNIs that are appropriate for chronic use in humans, such as nerve cuff electrodes, typically suffer poor recording selectivity. A novel method has recently been developed that uses multi-contact nerve cuffs to obtain spatiotemporal signatures of neural activity, which get fed into convolutional neural networks that can distinguish neural pathways with improved recording selectivity.

The purpose of this project was to evaluate the robustness of this approach to common forms of interference, such as muscle activity and stimulation artefact, and to variations in electrode geometry. Computational modeling and simulation of the rat sciatic nerve were used to generate synthetic neural recordings obtained with different electrode geometries (cylindrical cuffs, flat interface nerve electrodes), and with different forms of added interference. Preliminary results suggest that flat interface geometries may better extract neural signals than cylindrical cuffs, and that classification accuracy may be impaired by structured forms of interference.
Iteratively improving the performance of this method will increase its applicability to clinical scenarios, such as closedloop stimulation systems for rehabilitation.

\section{Improving Cellular Uptake of Lipid Nanopar- ticles Encapsulating siRNA}

\section{Sami Sabbah¹, Kai Slaughter ${ }^{1}$, Molly Shoichet ${ }^{2}$ \\ ${ }^{1}$ Institute of Biomedical Engineering, University of Toronto \\ ${ }^{2}$ Chemical Engineering \& Applied Chemistry, University of Toronto}

RNA interference (RNAi), which can selectively inhibit gene expression through sequence homology, is a promising approach to target undruggable disease-associated proteins. Lipid nanoparticles (LNPs) are a leading technology for siRNA delivery and were recently approved for clinical use as the drug Onpattro to treat amyloidosis. There has been recent interest to expand this therapeutic platform beyond hepatic sites to treat diseases such as cancer or autoimmune disorders. Here, we examine the impact of modifying the composition of LNPs encapsulating siRNA on cellular uptake and knockdown of a model gene in cancer cells. LNP formulations were prepared by microfluidic mixing and characterized in terms of hydrodynamic diameter (dynamic light scattering) and RNA encapsulation efficiency (RiboGreen assay). An initial formulation employing a standard lipid composition from literature produced LNPs with small size $(\sim 120 \mathrm{~nm})$ and high siRNA encapsulation efficiency (>90\%), but cellular uptake in cancer cells (measured by fluorescence microscopy) was limited. To improve uptake, the composition of non-bioactive components of the LNP formulations was modified. We observed that improved cellular uptake could be achieved through the selection of an appropriate phospholipid. However, even with increased cellular uptake, the knockdown of a model gene (firefly luciferase) was not observed, suggesting poor endosomal escape. Future work will focus on further optimization of LNP formulation components to improve endosomal escape and subsequent gene knockdown. These understandings can promote the development of RNAi-based therapies for previously difficult to treat conditions.

ScRNA-seq Analysis on TRACER Reveals Hypoxia-Specific Ligand Pair Interactions 


\section{between Pancreatic Cancer Cell and Macropha- ges}

\section{Ziting (Judy) Xia', Ileana Co², Alison McGuigan¹,2}

${ }^{1}$ Department of Chemical Engineering and Applied Chemistry, University of Toronto

${ }^{2}$ Institute of Biomedical Engineering, University of Toronto

Pancreatic ductal adenocarcinoma (PDAC) is a malignancy having the worst prognosis amongst all solid tumors, with a 5-year survival rate of only 7-8\%. Tumor-associated macrophages (TAMs) are a key immune cell type that infiltrates the tumor microenvironment (TME). Hypoxic TME reprograms TAMs to a pro-tumoral phenotype, contributing highly to such poor prognosis. However, the exact interactions between PDAC and TAMs under hypoxic influence have yet to be elucidated. The McGuigan Lab has developed an in vitro 3D PDAC-TAM co-culture system called TRACER (Tissue Roll for Analysis of Cellular and Environment Response), which recapitulates the hypoxic TME. We used single cell RNA sequencing (scRNA-seq), a powerful tool for dissecting tumor samples on a single cell resolution, to interrogate the PDACTAMs crosstalk. Co-cultured PDAC-TAM are digested from the TRACER scaffold and analyzed by scRNA-seq. Using the Seurat v3.0 pipeline, we are able to annotate heterogeneous tumor and macrophage cell populations and identify them as "Hypoxic" or "Normoxic" by calculating their hypoxic scores through hypoxic gene expression. The transcriptomic data of these annotated cell clusters are then input to CellPhoneDB, a cell-cell communication network that maps out possible ligand-receptor interactions between PDAC and TAMs. Preliminary results identified 102 hypoxia unique interactions and several hypoxic gene pairs that are highly consistent across different samples (ex. COL17A1-a2b1 complex for TME remodelling). Future work will be devoted to validating these interactions in vitro and testing efficacy of specific receptor antagonists. These findings will contribute to developing new combinational immunotherapy, bringing new hopes to patient with PDAC.

\section{Characterization of a co-electrospun nanofib- rous gelatin polyurethane composite scaffold for cardiac tissue engineering}

Willa Wei ${ }^{1}$, Yizhou Chen ${ }^{2,3}$, J. Paul Santerre ${ }^{2,3,4}$
${ }^{1}$ Department of Chemical Engineering and Applied Chemist- ry, University of Toronto

${ }^{2}$ Institute of Biomedical Engineering, University of Toronto

${ }^{3}$ Translational Biology and Engineering Program, Ted Rogers Centre for Heart Research

${ }^{4}$ Faculty of Dentistry, University of Toronto

Electrospun nanofibrous scaffolds are suitable for tissue engineering as their fibrous structure resembles that of native extracellular matrix (ECM). Previously, a degradable polar/ hydrophobic/ionic polyurethane (D- PHI) and polycarbonate polyurethane (PCNU) were generated, which showed good biocompatibility, however their fibre mesh remained too stiff ( $\sim 55 \mathrm{MPa})$ and degraded slowly (> 90 days) for the cardiac applications of interest. Gelatin improves hydrophilicity, alters mechanical properties, and are anticipated to enhance cell attachment when blended with synthetic polymers. A 55:20:25 gelatin/D-PHI/PCNU co-electrospun scaffold was generated in the Santerre lab and was hypothesized to take 1-2 months to degrade and have a reduced elastic modulus. The objective of this study was to investigate fibre distributions, fibre morphology, and mechanical properties of this scaffold as it degrades. Both crosslinked and uncrosslinked gelatin for the 55:20:25 composite and the original D-PHI/PCNU coelectrospun scaffolds were generated. The scaffolds' weight losses were recorded with respect to time when exposed to 0.013 units $/ \mathrm{mL}$ collagenase $A$, and/or 10 units $/ \mathrm{mL}$ of cholesterol esterase (CE) in PBS solutions at $37^{\circ} \mathrm{C}$. Scanning electron microscopy (SEM), differential scanning calorimetry (DSC), Fourier transform infrared spectroscopy (FTIR), and biaxial mechanical tests were performed. $56 \mathrm{wt} \%$ of the crosslinked gelatin/D-PHI/PCNU scaffold was lost by Day 1 due to the removal of gelatin indicated by FTIR spectra. The same material also lost $73 \%$ by Day 28 . CE catalyzed degradation showed gradual weight loss that reached $93 \%$ by Day 28 . These mass losses were not observed with D-PHI/PCNU controls. This demonstrated that the presence of gelatin accelerated the degradation of D-PHI and PCNU relative to literature findings. Mechanical test data will be available by late August. On-going work is assessing the in vivo compatibility related to material and degradation product interactions with human pluripotent stem cell derived cardiomyocytes. 


\section{A common postural control between reactive balance and quiet standing}

\author{
Joanna Li1, Kai Lon Fok ${ }^{2,3}$, Kei Masani2,3 \\ ${ }^{1}$ Division of Engineering Science, University of Toronto \\ ${ }^{2}$ Institute of Biomedical Engineering, University of Toronto \\ ${ }^{3}$ Kite Research Institute, Toronto Rehabilitation Institute, \\ University Health Network
}

Human bipedal stance is inherently unstable as a large mass is kept upright with its center of mass located high above a relatively small base of support. Continuous controls on leg muscles - including reactive and proactive balance controls - are critical in maintaining standing balance. It has been assumed that these controls are independent, with reactive control operating during mechanical perturbation and proactive control operating during undisturbed quiet standing. Conflicting findings have been described in studies regarding the relationship between reactive and proactive controls, which is likely because inconsistent perturbation methods applied to the body or standing platform induce different reactive behaviours. Nevertheless, a few studies have shown that a common control system can account for both behaviours. Therefore, the purpose of this study was to investigate whether a common control can explain the postural behaviour during quiet and perturbed standing. Eleven healthy young individuals participated in the experiment. Participants stood on a force platform and performed (1) quiet standing and (2) standing perturbed by a pull at the waist. When we applied a pinned polymer model to standing posture and the fluctuation-dissipation theorem, quiet standing behaviour predicted reactive balance behaviour. These results were replicated through simulations. We expect to obtain results supporting the hypothesis that a common control accounts for both reactive and quiet standing balance. These findings will be beneficial when considering efficient assessments and interventions for standing balance in individuals with deteriorated lower limb functions such as stroke survivors and elderly

\section{Morphology-based classification scheme for endocytic vesicles on electron microscopy ima- ges}

Wanda Janaeska1 ${ }^{1}$, Jamie L. Y. Wu ${ }^{2,3}$, Wayne Ngo ${ }^{2,3}$,
Warren C. W. Chan ${ }^{2,3,4,5,6}$

' Division of Engineering Science, University of Toronto

${ }^{2}$ Institute of Biomedical Engineering, University of Toronto

${ }^{3}$ Terrence Donnelly Centre for Cellular and Biomolecular Research, University of Toronto

${ }^{4}$ Department of Chemistry, University of Toronto

${ }^{5}$ Department of Chemical Engineering, University of Toronto ${ }^{6}$ Department of Materials Science and Engineering, University of Toronto

One of the mechanisms for nanoparticles to deliver imaging and therapeutic agents to tumours is through uptake and transport across the vascular endothelial cells. Currently, it is unclear which endocytosis pathways facilitate this process. The major pathways that may be involved include caveolae-mediated endocytosis, clathrin-mediated endocytosis, and macropinocytosis. Different uptake pathways have different vesicle types associated with them. Studying nanoparticle-containing vesicles using transmission electron microscopy (TEM) provides direct evidence to the involvement of these pathways. However, the morphologies of the vesicles have been challenging to differentiate on TEM images subjectively. To address this, we develop a classification algorithm for endocytic vesicles on TEM images to help study nanoparticle localization. We imaged tumour slices from mice using TEM, which has the resolution necessary to resolve these endocytic vesicles. Using Image and MATLAB ${ }^{\circledR}$, we quantified vesicle parameters, such as: cross-sectional area, circularity, contrast between lumen and vesicle coat, and outline thickness. For example, we found that clathrin-coated vesicles have $46 \%$ and $27 \%$ thicker coats than caveolae and macropinosomes, respectively. We built a semi-automated classification algorithm using measured features that are capable of distinguishing different vesicle types. Next, we need to validate said algorithm by comparing its results with subjective classification done by human judgement. Being able to characterize different vesicle types will help us classify nanoparticle- containing vesicles inside endothelial cells objectively and consistently. This will teach us the uptake pathway for nanoparticle delivery into tumours and inform design strategies that can increase nanoparticle delivery to cancer cells.

\section{Auditory Cues Modify the Fractal Dynamics of}




\section{Human Gait during Treadmill Walking in Male Participants}

\author{
T Marshall ${ }^{1,2,3}$, H Rouhani ${ }^{4}, \mathrm{M} O \mathrm{Abe}^{5}, \mathrm{~K} \mathrm{Na}-$ \\ kazawa ${ }^{6}$, D Nozaki ${ }^{7}, \mathrm{~K}$ Masani ${ }^{2,3}$ \\ ${ }^{1}$ Department of Engineering Physics, McMaster University \\ 2 Institute of Biomedical Engineering, University of Toronto \\ ${ }^{3}$ KITE - Toronto Rehabilitation Institute, University Health \\ Network \\ ${ }^{4}$ Department of Mechanical Engineering, University of Alber- \\ ta \\ ${ }^{5}$ Hokkaido University \\ ${ }^{6}$ Department of Life Sciences, University of Tokyo \\ ${ }^{7}$ Department of Physical Education, University of Tokyo
}

Stride interval (SI) fluctuations during human locomotion exhibit unique behaviour called fractal dynamics, which disappears when gait is accompanied by rhythmic auditory cues. Fractal dynamics are characterized by long-range correlations between seemingly random fluctuations in strides with significant temporal separations. An understanding of the mechanisms that produce fractal dynamics in gait is central to the development of models of the locomotor system. Here, we investigated the fractal dynamics of treadmill walking with a metronome. Ten healthy, young, male participants walked on a treadmill under two gait conditions: at their preferred pace (free walking, FW) and with a cue provided by a metronome (metronome walking, MW). As in previous studies, the detrended fluctuation analysis (DFA) plot for FW indicated the presence of fractal dynamics in SI series, while the plots for MW were "curved" at a window period of approximately 30 strides. We demonstrated through simulations that $\mathrm{SI}$ fluctuations during $\mathrm{MW}$ result from a combination of the participant's innate fractal dynamics and its deviation from the metronomic cue; DFA plots of the simulated time series, as in experimental MW, were nonlinear, supporting the hypothesized mechanism. DFA of the difference series of FW SI was then performed and compared to that of $\mathrm{MW}$ in each participant. We found that difference series with differencing periods of $6 \pm 2$ strides approximated the dynamics of $\mathrm{MW}$, suggesting that the participants used a stride approximately 6 strides in the past to correct the stride-metronome discrepancy. These results provide novel insights into human gait dynamics and will contribute to the development of more robust models and diagnostic tools.

\section{Data analysis of volatile organic compounds in Canada: sources, spatiotemporal trends and patterns related to volatile chemical products}

Urvi Verkhedkar, Amirashkan Askari, Arthur Chan

Department of Chemical Engineering and Applied Chemistry, University of Toronto

Volatile Organic Compounds (VOCs) are chemicals that evaporate readily at room temperature which contribute to air pollution by reacting to form ozone and particulate matter in the atmosphere. Over the past decades, VOC emissions from fossil-fuels have greatly decreased in North America with Volatile Chemical Products (VCPs)- such as pesticides, coatings, adhesives, inks, personal care products - now emerging as significant sources in cities in the United States. As VCP usage is not extensively regulated nor studied in Canada, there is a need to quantify their contribution to VOC emissions and study spatiotemporal and demographic emission patterns in Canadian cities. We hypothesize that VCPs are substantial emission sources that positively correlate with population density and time. Concentration data were collected from the Canadian National Air Pollution Surveillance (NAPS) database for 176 VOCs in 110 stations for 30 years across Canada. The data analysis methods constitute the following: Principal Component Analysis (PCA), a standard technique for source apportionment; boxplots to visualize general changes in data distributions over time; time series smoothing for detailed temporal modelling; regression analysis with population density and level of urbanization; and, results comparison for different provinces for spatial variation. Preliminary results indicate an increase in dichloromethane emissions across Canada from 1994-2019, suggesting VCP behaviour, although further analysis (PCA, regression) is needed for conclusive results. Understanding the extent and patterns of VCP emissions can help inform environmental policies in Canada and other nations on similar development trajectories, further improving urban air quality.

\section{Smart Polymer Coating to Minimize Caustic In- jury from Battery Ingestion in Children}

Ruiping Yang ${ }^{1}$, Jacky Liao², Deena Adamjee', Mahsa Karimi ${ }^{1}$ 
${ }^{1}$ Department of Material Science \& Engineering, University of Toronto

\section{Engineering Science, University of Toronto}

Caustic injuries caused by the ingestion of Button Battery (BB) has been a growing health hazard due to the spread of common consumer electronics. Over three thousand children in the US alone are hospitalized each year due to accidental battery ingestion. When a battery is swallowed it reacts with our bodily fluids in an electrolysis reaction resulting in tissue burns from the hydroxide ion build-up at the anode of the battery. Studies show that batteries should be removed within 2 hours of ingestion in order to reduce the chances of serious injury. Time, therefore, is a crucial factor to consider when dealing with battery ingestion. There are no commercial designs available that prolong the 2 hour "safe" period or insulate the BB when ingested. We aim to fill this gap by applying smart non-conductive biopolymer materials filled with biodegradable metal particles that react in specific conditions, such as $\mathrm{pH}$, to insulate the battery when accidentally swallowed. In the case of ingestion, the polymer would swell due to changing $\mathrm{pH}$ conditions, cutting off any voltage leakage. The design objective for this project is to develop and validate such design that can be implemented on a commercial scale. The validation process involves ensuring the effectiveness of such design. Potential metrics are the durability, longevity and reducing the cost of implementation. Current and future research would focus on a mixture that has a rapid reaction rate and stability in solid form.

\section{The Addition of Water to Heated Cooking Oils and the Effect on Gas and Particle Phase Emis- sions}

\section{Eleanor Vaz', Jenna Ditto ${ }^{2,3}$, Arthur Chan ${ }^{3}$ \\ ${ }^{1}$ Chemical Engineering, University of Toronto \\ ${ }^{2}$ Department of Chemistry, University of Toronto}

Traditional emission sources of air pollutants, such as vehicular traffic, are decreasing because of technological advancements in response to environmental regulations. However, volatile organic compound (VOC) emissions from other anthropogenic sources, like cooking, are becoming increasingly important. Cooking influences urban air quality and is a source of organic aerosol to the atmosphere. Respiratory irritants, like aldehydes, emitted from the thermal degradation of cooking oil pose a threat to human health. During cooking, food moisture released into oil can result in hydrolysis reactions, furthering oil degradation mechanisms and VOCs emissions. The project objective is to study these mechanisms to accurately predict the emission rates of VOCs from all cooking styles.

To study the effect of water on oil degradation, multiple ratios of cooking oil-to-water emulsions were heated in an insulated aluminum block housed in an acrylic box to simulate cooking in a controlled environment. Tenax tubes and quartz filters collected gas- and particle-phase emissions, respectively. Offline chemical analysis was performed on samples using thermal desorption with gas chromatography and mass spectrometry. Additionally, particle number concentrations and size distributions were obtained using an optical particle counter. Emissions from oil-water emulsions varied in the gas- and particle-phases and increased in particulate matter mass concentration compared to oil. The presence of water as a weak nucleophilic source in hydrolysis reactions influenced the concentration of carboxylic acids available for direct emission, or for other decomposition reactions that yield species like aldehydes. This has implications for improving cooking emission modelling in urban atmospheres.

\section{Microwave-Enabled Activation of Waste Tea Leaves For Supercapacitor Electrodes}

Tianyu Liư1, Raunaq Bagchi1,2, Keryn Lian,2

${ }^{1}$ Department of Materials Science \& Engineering, University of Toronto

\section{${ }^{2}$ Flexible Energy and Electronics Laboratory, University of} Toronto

Electrochemical capacitors (EC) are energy storage devices with higher energy density than electrolytic capacitors and higher power density than batteries, making them suitable for applications from electric vehicles to wearable electronics. ECs are composed of two electrodes physically separated and wetted by an ionic conducting electrolyte. Porous carbons are excellent electrode materials due to high specific surface area, good electronic conductivity and good chemical stability. Waste biomass feedstocks, such as corncobs and tea leaves, are abundant, sustainable, low cost, and can be used to produce porous carbon. Waste tea leaves have been 
carbonized at high temperatures to a carbon-rich biochar followed by a porosity generating activation, where chemical activation with $\mathrm{KOH}$ is commonly used. However, these processes involve high energy and material consumption, non-uniform heating and long processing time.

In this study, microwave-based activation using phosphoric acid on waste tea leaves biochar was investigated as an alternative approach to conventional chemical activation. The heating duration and char-to-acid ratio were varied to explore the optimum condition. Free standing electrode films made from the activated carbons were studied using electrochemical techniques, such as cyclic voltammetry. Surface morphology and chemistry of the activated carbons were investigated through Scanning Electron Microscopy (SEM), X-ray Photoelectron Spectroscopy (XPS) and Micro Raman spectroscopy. The proposed method will significantly reduce the length of the activation process, and is expected to maintain comparable performance to the conventional method. Future work can be conducted on one-step conversion from biomass to activated carbon through microwave heating.

\section{The Effect of Street Sweeping on Road Dust and PM Loadings in the City of Toronto}

\section{Eeman Abdulkadir ${ }^{1}$, Cuilian Fang ${ }^{1}$, Cheol-Heon Jeong ${ }^{1,2}$, Greg J. Evans ${ }^{1,2}$ \\ ${ }^{1}$ Department of Chemical Engineering, University of Toronto \\ ${ }^{2}$ Southern Ontario Centre for Atmospheric Aerosol Rese- arch, University of Toronto}

Road dust, consisting of particulate matter (PM), reduces air quality and can cause health issues related to the cardiovascular and respiratory systems. Therefore, the City of Toronto's Clean Roads to Clean Air Program is aimed at improving air quality by utilizing street sweeping to reduce the amount of road dust on city streets. Currently, the City is improving their sweeping program but is limited due to gaps in knowledge, including information on road dust accumulation trends and the long-term effect of sweeping on PM concentrations.

The purpose of this project is to identify governing parameters to support the development of operational guidelines for the City's sweeping program based on the influence of sweeping on air quality and road dust accumulation trends. To achieve this, Aerodynamic Particle Sizer (APS) data will be analyzed to determine the effect of sweeping on PM concentrations over time. In addition, onroad monitoring of PM loadings has been conducted weekly to collect data on accumulation trends.

Preliminary results from monitoring have revealed that dust loadings stay relatively constant on roads that are swept daily, while less swept roads show more variability from week to week. As APS data is analyzed, it is expected that findings will result in a decline of PM concentrations in the long-term as lower road dust accumulation will lead to less resuspension over time. Next steps include analyzing longterm PM concentration trends and improving the monitoring procedure to collect sufficient data that will support the City in the improvement of their program.

\section{Increasing biochar yield from the carbonisation of Polyolefin waste via pyrolysis}

\section{Ankita Kapoor, Charles Jia, Donald Kirk}

\section{Department of Chemical Engineering and Applied Science}

Canadians produce $\sim 3.3$ trillion tonnes of plastic waste annually, 53.3\% being polyolefins. Polyolefins, such as polyethylene and polypropylene, are polymers with alkene mer units. Only $9 \%$ of this waste is recycled, the rest is incinerated or sent to landfills, neither environmentally conscious. Pyrolysis can be used to upcycle this waste into biochar, enabling its use in supercapacitors or wastewater treatment applications. Pyrolysis of organic wastes, including polymers, has great potential as a negative emissions technology. Since pyrolysis takes place without oxygen, no $\mathrm{CO} 2$ is released, allowing the sequestration of carbon. Biochar breaks down slowly, capturing this carbon for long periods. Unfortunately, the linearity of polyolefins makes it difficult to carbonise directly. Pyrolysis of polyolefins causes gasification of most of the material, resulting in a meagre biochar yield $(\sim 2 \%)$ and the emission of $\mathrm{CO} 2, \mathrm{CO}$, and $\mathrm{H} 2$.

This study explores different types of polymer pretreatment available to stabilise polyolefins before carbonisation, in efforts to lower gasification and increase the yield of biochar to at least $50 \%$. Literature review was used as COVID hindered lab work. Some of the methods analysed include thermal oxidation, sulphonation, and bromination. Thermal oxidation is the most environmentally friendly, as it 
makes use of oxygen, but the least effective - with a $57.2 \%$ mass yield. Sulphonation makes use of sulphuric acid, making it less environmentally friendly, but has a biochar yield of $\sim 80 \%$. Bromination results in a yield of $57 \%$ but is also harmful to the environment. The methods will be further compared by looking at the overall environmental impact, efficacy, cost, and elemental composition of char. The effectiveness of the proposed methods require verification in the future, via lab work.

\section{Determining Optimal Geometric Parameters for 2-Terminal All Perovskite Tandem Solar Cells using an Artificial Neural Network Model}

\author{
Nick Nabavi ${ }^{1}$, Erik Birgersson², Xinhai Zhao², Han- \\ song Xue ${ }^{2}$, Hu Quee Tan² \\ ${ }^{1}$ Engineering Science, University of Toronto \\ 2 Solar Energy Research Institute of Singapore, National Uni- \\ versity of Singapore
}

In view of the worsening energy and environmental crisis, there is an urgent need to develop efficient renewable energy technologies. Given the precipitous increase in efficiency for perovskite-based solar cells, they are a uniquely promising renewable. In particular, 2-terminal all perovskite tandem solar cells (2T APTSCs) have considerable potential to surpass the Shockley-Queisser efficiency limit of single junction solar cells. However, a major roadblock in achieving this goal is determining optimal geometric properties of $2 \mathrm{~T}$ APTSCs. Although mechanistic models exist which capture the most important characteristics of $2 \mathrm{~T}$ APTSCs, they are very computationally taxing, much more so than Artificial Neural Networks (ANNs).

The purpose of this research project is to develop a fast and accurate ANN model for a 2T APTSC in order to determine which geometric parameters will lead to optimum power conversion efficiencies, using data provided by the Solar Energy Research Institute of Singapore. Accuracy of the ANN is measured using mean-squared error, with speed measured by training time. The MATLAB Deep Learning Toolkit is used to develop the framework for the ANN, and hyperparameters are optimized using Bayesian Optimization and Particle Swarm Optimization. More global optimization algorithms such as the genetic algorithm and simulated annealing can be used in the future to select the most accurate and fast neural network. With this ANN, efficiency optimization studies for $2 T$ APTSCs can be done rapidly. Hence, using slightly different datasets can allow for parameters other than geometric ones to be optimized as well.

\section{A Comparison of Analysis Methods for Unders- tanding Anthropological Emissions and Geos- patial Differences in Traffic Related NOx Emis- sions Due to the Holiday Effect in Ontario}

\author{
Karen Chen¹, Greg Evans², Vicky Chen³, Cheol- \\ Heon Jeong'2, Grace Liu ${ }^{4}$ \\ 1 Engineering Science, University of Toronto \\ ${ }^{2}$ Southern Ontario Centre for Atmospheric Aerosol Research \\ ${ }^{3}$ Applied Statistics, University of Toronto \\ ${ }^{4}$ Department of Arts and Sciences, University of Toronto
}

Nitrogen Oxides (NOx) are one of the most abundant primary air pollutants, frequently formed from the combustion reactions between nitrogen and oxygen during vehicle exhaust production. NOx is a prominent oxidizing agent that reacts with volatile organic compounds to produce ground-level ozone and fine particulate matter (PM), which poses a detriment to human respiratory health. Therefore, developing a comprehensive knowledge of NOx trends and sources is paramount to examining potential policy changes or human activity in improving air quality. Assessing significant timelines such as annual holidays is an effective way to isolate the effect of anthropological emissions - specifically derived from transportation patterns. However, a comparison of published literature alongside preliminary analysis between three pollutant-concentration calculation methods indicates a significant inconsistency in reported magnitudes of change within identical datasets. Furthermore, few studies have examined North American trends, and none have been conducted in Canada. The goal of this research moving forward is to develop a more comprehensive analysis method in capturing the impact of factors including seasonality and datetime matching on pollution-concentration change outputs. Specifically, the analyses will focus on annual winter holiday data (from mid-December to early January), when traffic emissions escalate due to increased travel. Geospatial sorting of results is hypothesized to demonstrate a greater increase of NOx concentrations surrounding major roadways and industry; however, the accuracy of these magnitudes may 
distinctly vary depending on the accuracy of each examined method's ability to identify nuances in human behavior, temporal, and seasonal conditions which govern fluctuations in NOx measurements.

\section{Numerical Approximation of the Schrödinger Equation}

\section{Alison Okumura ${ }^{1}$, Fadime Bekmambetova², Piero Triverio ${ }^{2}$ \\ ${ }^{1}$ Engineering Science, University of Toronto \\ ${ }^{2}$ Department of Electrical and Computer Engineering, Uni- versity of Toronto}

Finite-Difference Time-Domain (FDTD) technique is a numerical approximation used in electromagnetism to solve Maxwell's equations. It uses central differences to discretize derivative operators and develop update equations that may be used iteratively to form solutions to a system. Such a tool is relevant as it can numerically model systems without analytical solutions to provide understanding of physical systems. This understanding can be applied to the development of quantum devices such as quantum dots and electronic components in nano size scale. As such, it is well suited to be extended to Schrödinger's equation. While FDTD technique is well explored in the domain of electromagnetism, the parameters of the quantum implementation (FDTD-Q) are not as consistently stated in literature. This investigation determines numerical stability and accuracy of FDTD-Q using MATLAB while verifying the results in literature. To achieve this goal, the project will implement the FDTD-Q equations in an iterative manner to compare the results against current literature and known analytical solutions. Such a comparison will provide an analysis on the stability and accuracy of such methods and identify any discrepancies in existing literature. We have identified behaviours of simulations operating around the time-step stability limit that differ from literature. Moreover, we have examined numerical accuracy in function of grid refinement - spatial-step size - using known analytical systems like an infinite well. Next steps take this project to the Maxwell- Schrödinger coupled system which will take FDTD-Q schemes from literature and analyze their relevance in modelling electron behaviour in an electromagnetic field.

\section{An Evaluation of the Translation of Cellular Response Prediction (TCRP) Few-Shot Learning Model for Prediction of Drug Response Between Preclinical and Clinical Contexts}

\section{Katherine Zhu ${ }^{1}$, Petr Smirnov 2,3,4,5, Benjamin Hai-} be-Kains $2,3,4,5,6$

\section{Division of Engineering Science, University of Toronto}

2 Princess Margaret Cancer Centre, University Health Network

\author{
${ }^{3}$ Department of Medical Biophysics, University of Toronto \\ ${ }^{4}$ Vector Institute \\ ${ }^{5}$ Department of Computer Science, University of Toronto \\ ${ }^{6}$ Ontario Institute for Cancer Research
}

Large-scale cell line pharmacogenomic databases have allowed for the development of computational models that provide an individualized prediction of drug response. However, due to the limited availability of data and the added complexity of clinical settings, it has proven difficult to translate these models from preclinical to human-tumour domains. Transfer learning, a machine learning method through which knowledge from a previous context is applied to the learning of a new and related situation, is a promising approach in overcoming these barriers to translation between domains. Among others, a few-shot transfer learning model titled "Translation of Cellular Response Prediction (TCRP)" has been proposed for translation between different settings, with reported significant performance improvement over other machine learning models. In TCRP, the model is pretrained on an abundance of samples obtained from cell lines, before being exposed to a small number of samples from a new but related context in a few-shot training phase. Model performance is then measured through its accuracy in testing on unseen samples from the new context. The purpose of this research is to critically evaluate the potential of TCRP as a predictive model for overcoming barriers in the translation of drug response among preclinical and clinical domains. This is done through a comparison of the model's performance against other baseline machine learning algorithms, notably Random Forest, K-Nearest Neighbours, and Elastic Net. In initial investigations, model performance was evaluated through its accuracy, measured with Pearson's correlation, in predicting drug response in cell lines of an unseen tissue type. Preliminary results demonstrated that TRCP does 
not significantly outperform the simpler machine learning significantly outperform the simpler machine learning methods. Nevertheless, future investigation of model performance in transfer learning contexts between cell lines and patient-derived tumour cells will be conducted. This will allow for further evaluation of the model's transferability towards patient settings, as the greater complexity of patientderived tumour cells more closely replicates the ultimate purpose of translating results towards clinical domains.

\section{Validation of 12-Bit 600 nV-Offset Buffered DAC for Analog Resistive Switching Memory Based Vector- Matrix Multiplication (VMM) Engines for Accelerated Machine Learning}

\author{
Junrui Zhang ${ }^{1}$, Jianxiong $\mathrm{Xu}^{2}$ \\ ' Engineering Science, University of Toronto \\ 2 Electrical \& Computer Engineering, University of Toronto
}

Using memristor crossbar arrays to efficiently complete massively parallel multiply and accumulated (MAC) operations is a promising method to achieve energy-efficient and accelerated machine learning according to A.P. Jame's studies of memristor-CMOS chips for Al. Most existing works use low-resolution circuits for small-sized arrays or off-chip high-resolution digital to analogue converter DAC, which is recognized as a gap in the current research field because this limits the performance of large crossbar arrays. The goal of this project is to develop and validate a high-resolution robust DAC that can accurately provide different ranges of voltage to support inference, reading, programming and electroforming to improve the performance of resistive switching memorybased vector-matrix multiplication (VMM) engines.The proposed 12-bit 600nV-Offset Buffered DAC developed by the ISML innovatively uses a ping-pong auto-zero (PPAZ) buffer which is capable of providing large inference current, and precise programming voltage with a broad tolerance of load resistance. The validation process requires designing a PCB and an FPGA test-board to drive the DAC with the requirements of reducing noise and clock skew. The goal of the validation process is to verify three things: the hypothesis that PPAZ can drive small resistive loads as well as robustly suppress the offset and the flicker noise; the DAC has an expected result of $11 \mathrm{~m} \Omega$ output impedance, $96 \mathrm{~dB}$ spuriousfree dynamic range (SFDR), $<600 \mathrm{nV}$ voltage offset and a sub-
$\mathrm{Hz}$ flicker noise corner frequency; and the proposed VDAC will dissipate power as low as $351.12 \mathrm{uW}$ from a 3.3V supply.

\section{An Effective and Automated Benchmark Sys- tem: Enabling the Adoption of a New Autono- mous Driving Dataset}

\section{Andrew Zou Li ${ }^{1}$, Keenan Burnett ${ }^{2}$, Tim Barfoot ${ }^{2}$ \\ 1 Division of Engineering Science, University of Toronto \\ 2 University of Toronto Institute for Aerospace Studies}

Researchers use autonomous driving datasets and accompanying benchmarks to obtain quality feedback for tasks such as vehicle odometry and object detection. An automated system is required to handle benchmarking for the University of Toronto's new Boreas All-Weather Autonomous Driving Dataset. The objective of this benchmarking system is to enable widespread adoption of this dataset and help researchers develop safer and more accurate models for self-driving. Primarily, the system seeks to maximize the ease and reliability of submission to benchmarks, assessed by the frequency of errors and speed of the process. Through serverless cloud computing, the benchmark system automatically handles user actions and executes benchmark evaluation scripts on valid uploads. Afterwards, the system updates a leaderboard database and sends results to users via email. The current serverless solution scales to any user pool size without the need for server maintenance and the entire process performs around 5 times faster than existing major datasets. Secondarily, the system will provide benchmark information for LiDAR object detection tasks by evaluating the difficulty of sequences within the dataset. To create a baseline for detection tasks, the average detection confidence from PointPillars will be used to estimate the difficulty of accurate detections on a given sequence. This metric provides more accurate categorization of sequence difficulty for benchmarks than the usual human-selected method. Once the dataset is near completion, future work on the benchmark system will be directed towards improving user experience and further testing the submission process to assess the failure rate.

Some (Team) Assembly Required: An Analysis of Collaborative Computer-Aided Design Assembly 


\section{Kathy Cheng, Alison Olechowski}

Mechanical and Industrial Engineering, University of Toronto

Previous efforts in collaborative computer-aided design (CAD) suggest that a team of designers working synchronously in a multi-user CAD (MUCAD) environment can produce CAD models faster than a single user. Our research is the among the first to investigate assemblies in MUCAD. In our study, 20 participants were tasked with assembling pre-modelled CAD parts of varying complexity in teams of one, two, three and four. We analyze audio recordings, team activity, and survey responses to compare the performance of individuals and virtual collaborative teams during assembly, while working with the same MUCAD platform.

This research features a multimodal approach to analyze team trends in communication, workflow, task allocation and challenges to determine which factors are conducive or detrimental to the success of a MUCAD team. In our work, the success of a team is measured by its productivity score, which is the number of mates added by a team within a given time frame. We present evidence that teams can complete an assembly in less calendar time than a single user, but single users are more efficient in person-hours, due to communication and coordination overheads. Surprisingly, paired contributors exhibit an assembly bonus effect. These findings represent a preliminary understanding of collaborative CAD assemblies. Our work supports the claim that collaborative assembly activities have the potential to improve the capabilities of modern product design teams, delivering products faster and at lower cost. We identify areas for future research, and highlight areas of improvement for collaborative CAD platforms and engineering design teams.

\section{What's Wrong With CAD? Identifying and Clas- sifying Challenges in Collaborative Work with Computer-Aided Design}

Michal Davis ${ }^{1}$, Jasmine Zhang ${ }^{1}$, Alison Olechows$\mathrm{ki}^{2,3}$, Shurui Zhou ${ }^{4}$

\section{${ }^{1}$ Division of Engineering Science, University of Toronto \\ 2 Department of Mechanical and Industrial Engineering, University of Toronto}

${ }^{3}$ Troost Institute for Leadership Education in Engineering, University of Toronto ${ }^{\circ}$ Department of Electrical and Computer Engineering, University of Toronto

${ }^{4}$ Department of Computer Science, University of Toronto
Computer-aided design (CAD) plays a key role in oftencollaborative hardware design processes. Despite the abundance of tools for collaboration with CAD, there is limited research clarifying the key challenges these tools must address. We aim to close this academic-practitioner gap through a classification of the challenges and their current responses in collaborative workflows involving CAD. Employing qualitative methods to address lack of existing theory, we conduct a four-phase study: literature review, classification development, interviews, and coding. An initial focused literature review is used to identify known challenges in both academic and practitioner writing. From this, we create the structure of our classification, consisting of pain points, their causes, mitigating strategies, and their flaws. In the semi- structured interview phase, five CAD professionals discuss the challenges they face when collaborating, with more participants expected as the research continues. We code the transcripts of these interviews in an opencoding process using the software NVivo, building on the classification developed in phase two. Currently, the classification contains thirteen pain points with twenty causes. Six mitigating strategies imperfectly resolve a subset of these pain points, with eighteen flaws between them. Several of these items are not found in the literature review, indicating potentially previously unknown challenges. This classification presents a guide for pressing areas of future work in CAD development. Future phases of this project will explore software development collaborative practice, where well-studied and broadly applied best practices and strategies for collaboration challenges present possible solutions to those identified here.

Floating or Drowning? Using Natural Language Processing Techniques to Understand Engineering Students' Learning Motivation in Relation to Workload Patterns in the Curriculum

Madison Mindorff1,2, Elnaz Baradaran Shokouhi1,2 Terence Chan ${ }^{1,3}$, Brendan Lobo ${ }^{1,2}$, Chirag Variawa ${ }^{1}$, Qin Liu ${ }^{1}$

${ }^{1}$ Institute for Studies in Transdisciplinary Engineering Education \& Practice, University of Toronto

${ }^{2}$ Department of Mechanical \& Industrial Engineering, University of Toronto

${ }^{3}$ Department of Statistical Sciences, University of Toronto 
Limited research has been done to examine the relationship between the motivation of engineering students and the typically heavy workload in engineering curriculum. Our previous study used sentiment analysis and text analytics to analyze students' weekly discussion responses for APS100, a mandatory first-year engineering course. The purpose was to understand the motivation levels of first-year engineering students in the virtual learning environment of the Fall 2020 term. To extend this work, this study uses natural language processing (NLP) as a method of analysis to explore how student motivation can relate to academic workload. For this research, 11763 student discussion board responses are exported from the course's online learning platform and are analyzed using Microsoft Azure's opinion mining and sentiment analysis features to identify student motivation. The levels of motivation are identified based on the expectancy-value theory. After the analysis, a second NLP model using an open-source architecture is used to validate the results. The levels of student motivation are then compared to a workload chart which includes the weights and deadlines of all first-year engineering assessments in the Fall 2020 term. It is expected that lower levels of motivation correlate with weeks that have multiple/major assessments and vice-versa. The results will establish whether NLP is an appropriate method that can determine the relationship between motivation and workload. If a relationship is found, these results can be used to support future changes that remove curriculum components shown to decrease student motivation. 